\title{
Los ciclos políticos en México, 1960-2011*
}

\author{
Miguel Cervantes Jiménez, ** Pablo López Sarabia*** \\ y Pedro Alejandro García Sámano****
}

\section{RESUMEN}

La presente investigación tiene por objetivo dilucidar la existencia, en el periodo de 1960 a 2011, de un ciclo político en la economía mexicana, que permita a los gobiernos en turno influir en variables económicas estratégicas durante los procesos electorales, incidir en la decisión de los electores y mantener el poder. Las variables utilizadas fueron el PIB real, el gasto público, la base monetaria y la tasa de inflación, con una frecuencia trimestral. Se estimaron dos modelos econométricos, uno por el método de mínimos cuadrados ordinarios y otro a través de vectores autorregresivos, para dicho periodo, aunque se construyeron dos subperiodos (1960-1983 y 1984-2011) debido al sobreendeudamiento que registró México en 1982. Los resultados en ambas metodologías sugieren la presencia de tal ciclo político en la economía, aunque los efectos son más significativos en el primer lapso. Se esboza la hipótesis de que los efectos son más débiles en el segundo porque la alternancia política ha sustituido la posibilidad de un impacto general por uno más focalizado en determinados estados y municipios. Finalmente, se presenta una propuesta de modificación al artículo 41 de la Constitución de los Estados Unidos Mexicanos, a fin de eliminar las distorsiones económicas que provocan los políticos en tiempos de elecciones.

Palabras clave: elección pública, ciclo político, política fiscal, elecciones, modelo econométrico.

Clasificación JEL: E61, E32, E62, E65, C01.

\section{Abstract}

The present investigation has the aim to prove the existence of a political cycle in the Mexican economy for the period from 1960 to 2011, which could allow the current government to affect strategic economic variables during elections in order to impact the economic cycle and have influence over the voters' decision and keep power. The variables that were used are the real GDP, public spending, the monetary base and the inflation rate on a quarterly basis. Two econometric models were estimated, one for the ordinary least squares method and the other through autoregressive vectors for this period, although two subperiods were built (1960-1983 and 1984-2011) due to the over-indebtedness recorded by Mexico in 1982. The results in both methodologies suggest the presence of a political cycle in the Mexican economy, although the effects are more significant in the first period; we assume that in the second period, the effects are weaker because the political alternation has reduced the possibility of an overall impact, for a more focused one in certain states and municipalities. Finally, we present a proposal to amend Article 41 of the Constitution of Mexico, in order to remove economic distortions that politicians cause at election time.

Keywords: public election, political cycle, fiscal policy, elections, econometric model. JEL classification: E61, E32, E62, E65, C01.

* Fecha de recepción: 17/05/2012. Fecha de aprobación: 03/09/2013.

** Jefe del Departamento de Teoría Económica, Facultad de Economía de la Universidad Nacional Autónoma de México. Correo electrónico: miguelc@economia.unam.mx.

*** Profesor-investigador del Instituto Tecnológico y de Estudios Superiores de Monterrey, campus Santa Fe. Correo electrónico: plsarabia@gmail.com y plsarabia@itesm.mx.

**** Asistente de investigador, Facultad de Economía de la Universidad Nacional Autónoma de México. Correo electrónico: alejandro.garsam@yahoo.com.mx. 


\section{INTRODUCCIÓN}

La crisis de deuda soberana y los desbalances fiscales que han experimentado las economías de la eurozona en los últimos años han obligado a países como Grecia, Portugal, Irlanda, España e Italia a instrumentar severos programas de ajuste fiscal, lo cual ha despertado la irritación de sus poblaciones ante los excesos en el gasto público en que incurrieron los respectivos gobiernos a fin de mantener el Estado de bienestar, que ha permitido que los ciudadanos tengan una percepción positiva sobre sus gobernantes y, con ello, su continuidad en el poder. En el caso de algunas economías de Sudamérica, el gasto público se ha utilizado como un instrumento para influir en el ciclo económico de corto plazo a fin de que los votantes tengan una opinión favorable del gobierno en turno. En el caso de México, el sobreendeudamiento de algunos estados y municipios ha sido cuestionado, ya que muchos de sus recursos han sido utilizados con fines electorales, reavivando la discusión sobre el uso del gasto público con objetivos partidarios.

Buchanan (1960), reconocido como fundador de la moderna teoría de la elección pública, desarrolló algunos de los elementos más conocidos de la relación entre economía y procesos electorales. Al respecto, Hotelling (1929) analizó el sistema del votante mediano, estableciendo que siempre que todas las preferencias de los individuos sean unimodales, el resultado mayoritario reflejará las de dicho votante, ya que se encuentran en la media del conjunto de las preferencias de todos los individuos. Por su parte, Lindahl (1958) determinó la tasa impositiva en el intercambio entre dos tipos de contribuyentes: ricos y pobres, en una situación en la que cada grupo paga un coste marginal igual a su valoración marginal del bien público. Otro autor importante fue Kalecki (1943), quien analizó la opción de financiar el pleno empleo a través de incrementos considerables del gasto público como política económica en tiempos de crisis. Años después, Samuelson (1954) argumentó que un bien público difiere de un bien privado porque el consumo del primero por parte de un individuo no reduce el consumo simultáneo de los demás; agregó que el costo marginal de suministrar un bien público es cero (casi siempre), ya que la exclusión de los individuos que no pagan es imposible.

Según Bonilla y Gatica (2005), en la actualidad la teoría de la economía política neoclásica se ocupa del paradigma de la elección racional, de acuerdo con el cual la acción política, individual o colectiva, responde a la aplicación de la racionalidad. Tres modelos la integran: la teoría de juegos y de información asimétrica, la teoría espacial del voto y la teoría del ciclo político-económico. La 
primera aborda el problema que surge cuando un agente individual interactúa con otros y el resultado final en ocasiones no es el que los agentes deseaban. Esta teoría se basa en el concepto de equilibrio de Nash y sus refinamientos hacia el equilibrio perfecto en subjuegos; ambos métodos de análisis de la teoría de juegos surgen de la heterogeneidad de los agentes respecto a sus intereses y objetivos, dado que no siempre son compatibles. La segunda estudia la manera en que las coaliciones gobernantes intentan retener el poder, además del hecho de que las características de los candidatos influyen en los resultados electorales, así como las causas y consecuencias de la incertidumbre en las votaciones. La tercera teoría explica que los ciclos económicos son manipulados por los políticos en tiempos electorales para conseguir su reelección.

En este tenor, este artículo tiene como objetivo verificar la presencia de un ciclo político en la economía mexicana durante el periodo de 1960 a 2011, por lo que la hipótesis que se plantea es que los gobiernos en turno buscan influir en variables económicas estratégicas como el gasto público y la base monetaria para incentivar el crecimiento del producto interno bruto (PIB) durante los procesos electorales e incidir en la decisión de los ciudadanos para mantener el poder (aunque un sobrecalentamiento de la actividad económica debería reflejarse en mayores presiones inflacionarias, situación que estaría alertando de que la economía se acerca a su nivel potencial). En el primer apartado se ofrece una introducción a la teoría de los ciclos políticos, con especial énfasis en la teoría de la elección pública; el segundo hace una revisión de la literatura, destacando la evidencia empírica; enseguida, en la tercera parte, se presentan los detalles de los modelos econométricos y las variables utilizadas para su estimación en el periodo de 1960-2011, así como los principales resultados empíricos; en el cuarto apartado se presenta una propuesta de carácter legal con el fin de acabar con las distorsiones negativas que provocan los políticos en tiempos de campaña electoral. Finalmente, se presentan las conclusiones de la investigación, en las que se confirma la presencia de un ciclo político en México, aunque con un impacto cada vez menor, debido a que la alternancia en el poder ha reducido la posibilidad de un efecto general y lo ha focalizado algunos estados y municipios.

\section{TEORÍA DE LOS CICLOS POLÍTICOS}

La teoría de los ciclos políticos es una rama de la teoría de la elección pública que define al ciclo económico como político porque las fluctuaciones de la economía son provocadas por los gestores de la política económica cuando influyen 
en algunas variables como el gasto público y la oferta monetaria, entre otras, con la intención de apaciguar temporalmente a la sociedad en tiempos de elecciones y hacerle creer que realizan un buen trabajo en pro de ella y así conseguir que los ciudadanos voten por su reelección o la permanencia del partido político en el poder.

Según la teoría de los ciclos políticos, tras unos comicios, el nivel de producción mantiene sus fluctuaciones en un nivel bajo, para crecer antes y durante los tiempos de la siguiente campaña electoral hasta pocos meses después de las elecciones. Posteriormente, en sus primeros meses de gestión, el nuevo gobierno, independientemente de que sea dirigido por el mismo partido político o por otro, busca reducir los altos niveles de gasto público, así como las demás variables económicas que fueron estimuladas por los gestores de política económica para las elecciones pasadas. Esto, con la finalidad de aminorar las altas tasas de inflación y para financiar el déficit resultado del incremento del gasto público, mediante el aumento de los impuestos, de la deuda pública y/o con la reducción del propio gasto público. Este proceso es cíclico y se repite cada vez que se acercan unos comicios.

Al considerar el comportamiento de los políticos y de los votantes, William D. Nordhaus (citado por Massad y Pattillo, 2000) clasificó a estos últimos en racionales y no racionales. A los partidos políticos los divide en oportunistas e ideológicos. A los primeros sólo les interesa llegar al poder o conservarlo y no les preocupan los resultados de sus políticas en la economía. Los segundos buscan alcanzar sus objetivos económicos y sociales minimizando la pérdida de bienestar de la sociedad; ganar las elecciones se deja en segundo plano.

La literatura reconoce cuatro modelos de ciclos políticos: a) partidos oportunistas con votantes no racionales, cuyo resultado es un modelo de ciclo oportunista; b) partidos ideológicos con votantes no racionales, relación de la que se obtiene un modelo de ciclo ideológico; c) votantes racionales, que hacen imposible la existencia de un ciclo político en la economía, y d) perturbaciones externas al proceso político, es decir, ya sea que los votantes sean o no racionales y tengan información incompleta o completa, puede suceder que no detecten el origen de los impactos que los afectan y la probabilidad de que se presente un ciclo político en la economía dependerá del tipo de partido en el poder.

Actualmente, la teoría propone al menos cuatro hipótesis sobre las motivaciones que subyacen en la conducta de los votantes, a saber: a) Racionalidad colectiva, es decir, que el comportamiento del votante se determina de acuerdo con su nivel de bienestar social. b) Votantes miopes, que son aquellos que se dejan 
llevar únicamente por el comportamiento de la economía durante los meses recientes antes de las elecciones y para quienes el nivel de la actividad económica es la variable determinante. c) Votantes ilusionados con el efecto del crecimiento del empleo, para los cuales la variable clave es el nivel de ocupación y no el de la inflación. d) Sesgo deficitario, esto es, cuando los políticos buscan crear más empleos incrementando el gasto público sin importar que éste exceda los ingresos que tiene el gobierno, pues el objetivo es ganar más votos. A continuación se reseña alguna de la literatura que aborda la evidencia empírica de los ciclos políticos.

\section{REVISIÓN DE LA LITERATURA: EVIDENCIA EMPÍRICA}

La literatura sobre la evidencia empírica del ciclo político es abundante, aunque existen algunos artículos que se han convertido en un referente. Por ejemplo, el de Nordhaus (1975) intitulado "The Political Business Cycle", donde analizó el problema de la elección entre inflación y desempleo utilizando el modelo mejorado de la curva de Phillips con expectativas adaptativas para explicar que los ciclos económicos son provocados por el comportamiento oportunista del gobierno en turno. Dicho autor concluye que el gobierno manipula la política económica, especialmente la política monetaria, antes de las elecciones para maximizar su probabilidad de reelección; al pasar las votaciones inicia una política de austeridad en la economía, provocando que se incremente el desempleo y disminuya la inflación. Esto sucede con cada elección, originando un ciclo político.

Hibbs (1977), en su documento "Political Parties and Macroeconomic Policy", expone dos trabajos con los que analizó la relación entre desempleo e inflación en lo referente a la orientación política de regímenes de 12 países de Europa occidental y de dos naciones norteamericanas. En su primer trabajo concluyó que la preferencia de los gobiernos de izquierda es de desempleo relativamente bajo a expensas de altas tasas de inflación; lo contrario sucede con los partidos de derecha. En el segundo demostró que los efectos de las políticas macroeconómicas de los gobiernos sobre las tasas de desempleo son asimilados más rápidamente en el sistema británico, ya que el ambiente político y económico de Estados Unidos es más descentralizado y heterogéneo que el de Reino Unido. Por ello, el nivel de desempleo se reduce con los gobiernos demócratas y se eleva con los gobiernos republicanos o conservadores. Lo anterior dio origen a la teoría del ciclo político económico partidista de Hibbs.

Tufte (1978), en su libro Political Control of the Economy, clasifica a los partidos políticos en dos tipos: izquierda y derecha, partiendo de que este último 
es el que detenta el poder hasta que lo pierde. Los partidos de izquierda se interesan por una distribución del ingreso más equitativa, por disminuir el desempleo y por incrementar el gasto del gobierno aceptando a la inflación y el futuro déficit fiscal, mientras que los de derecha prefieren lo contrario. Sobre el caso de Estados Unidos, afirma que en el periodo 1948-1976 se hicieron presentes dos tipos de ciclo, de acuerdo con la variable de estudio: uno consistió en un periodo de dos años con crecimiento del ingreso real disponible per cápita, aceleración durante otros dos años y desaceleración en años sin comicios, y el otro fue un ciclo presidencial de cuatro años reflejado en una disminución de la tasa de desempleo en los meses precedentes a la elección y un aumento en dicha tasa de 12 a 18 meses después de las elecciones.

Alesina y Roubini (1992) analizaron el comportamiento de la inflación, el crecimiento del PIB y el número de desempleados de 18 países de la Organización para la Cooperación y el Desarrollo Económico (OCDE) en el periodo de 1960 a 1987; además, utilizaron una variable cualitativa (dummy) para captar el efecto de las elecciones. Estos autores concluyen que, a excepción de Alemania y Nueva Zelanda, no se encontró prueba de un ciclo oportunista como el propuesto por Nordhaus, aunque los datos indican un ciclo electoral relacionado con la tasa de inflación. La mencionada teoría partidista de Hibbs es generalmente rechazada; una semejanza entre varios países es que los gobiernos de izquierda al ser elegidos estimulan la economía alrededor de dos años, después las expectativas de la inflación se ajustan y la economía regresa a su nivel de crecimiento natural; por el contrario, los gobiernos de derecha luchan contra la inflación, lo que origina una recesión y tiempo después la economía regresa a su nivel natural de crecimiento y la inflación se mantiene baja.

Téllez (1997), en su tesis sobre el ciclo político en México durante el periodo 1970-1994, analizó las siguientes variables: PIB, ingreso nacional disponible, inflación, población ocupada total, base monetaria, agregado monetario M1, gasto público (gasto programable y gasto neto devengado tanto del sector público como del gobierno federal). En su trabajo afirma que se cumple la hipótesis de la teoría del ciclo político de la economía, ya que las variables mencionadas crecen en el tercer y sexto años de elecciones, mientras que en el primer año del gobierno en turno se aplica una política de austeridad para estabilizar la economía.

Magaloni (2000) realizó varios modelos considerando distintos periodos de acuerdo a la información disponible de cada variable de estudio y se apoyó en una variable cualitativa para medir el efecto de las elecciones. En relación con la tasa de inflación y la base monetaria, analiza los periodos de 1970 a 1982 y de 
1982 a 1997. Los resultados indican que en la primera etapa los gobiernos estimularon la economía por medio de una expansión de la base monetaria, causando un alza en la inflación en años electorales, especialmente durante los comicios presidenciales de 1976 y 1982, mientras que en la segunda trataron de controlar la inflación antes de las elecciones, especialmente en los años 1988, 1991 y 1994. En lo que toca al gasto programable gubernamental en el periodo 1983-1998, los resultados muestran un crecimiento previo y posterior a las votaciones. Sobre el tipo de cambio para los años 1976-1997, los datos indican que las devaluaciones de la moneda se relacionan con el ciclo electoral, ya que éstas se presentaron en 1976, 1982, 1985 y 1994. Magaloni utiliza un último modelo para el crecimiento económico de 1980 a 1997, explicado por el gasto público. Sus resultados indican que hay un impulso en el crecimiento, así como en el consumo, en el trimestre previo a los comicios y que los gestores de la política lo estimularon antes de las elecciones y pospusieron la contracción de la actividad económica después de ellas.

Grier y Hernández-Trillo (2004), en su trabajo titulado "The Real Exchange Rate, Process and its Real Effects: The Cases of México and the USA", estudiaron el tipo de cambio real y la producción en las economías de México y de Estados Unidos en el periodo 1970-1998; también usaron una variable cualitativa para medir el efecto de las elecciones. El trabajo concluye que en México, durante dicho lapso, las elecciones afectaron significativamente la evolución del tipo de cambio real y que entre más se aprecie éste es menos predecible; a su vez, la depreciación del tipo de cambio real disminuye en gran medida la producción y la incertidumbre. En contraste, ninguno de estos resultados se presentó en la economía de Estados Unidos.

Gámez (2004) analizó la economía mexicana en el periodo 1980-2004 utilizando las tasas de crecimiento del PIB, el indicador coincidente, ${ }^{1}$ y el Índice Nacional de Precios al Consumidor, así como dos variables cualitativas para medir el efecto de las elecciones. Los resultados indican que en el año previo a éstas no crece el PIB ni la inflación, pero en el año posterior se presenta una recesión económica.

\footnotetext{
${ }^{1}$ El indicador coincidente (IC) para México es un índice compuesto que incluye información sobre los mercados de bienes, servicios y laboral. Dicho indicador incorpora el PIB mensual, el índice de volumen físico de la actividad industrial, el número de asegurados permanentes del Instituto Mexicano del Seguro Social, el índice de ventas al menudeo, las remuneraciones totales pagadas en la industria maquiladora de exportación y la tasa de ocupación parcial y desocupación (TOPD) (Gámez, 2004, p. 5).
} 
Flores (2007) separó las elecciones en presidenciales (cada seis años) y federales (cada tres años), de 1983 a 2005, con las siguientes variables de análisis: PIB real, gasto público real, tasa de desempleo, tasa de inflación, valor de la producción de Estados Unidos y la variable dicotómica "elecciones". Este autor concluye que ninguno de ambos comicios afecta el crecimiento del PIB ni en México ni en Estados Unidos y que carecen de influencia en el gasto público y el desempleo.

Gámez y Amarillas (2011) encuentran evidencia de un ciclo políticopresupuestal, tanto a nivel federal como en las entidades federativas de la República Mexicana, en el periodo de 1988 a 2010. Señalan que existen incrementos significativos en la tasa de crecimiento del gasto público, superiores al promedio registrado en los últimos meses de cada gobierno, seguidos por contracciones del gasto en los primeros meses de la nueva administración. En el siguiente apartado se presentan los modelos econométricos para medir el ciclo político en México.

\section{Modelo De CIClo POLÍTICO EN MÉXICO}

Si bien en la evidencia empírica reseñada se usan diferentes variables económicas para detectar la presencia de un ciclo político, en el presente estudio la modelación se aboca a cuatro variables económicas: el PIB y la inflación como variables respuesta y el gasto público y la base monetaria como variables instrumento de las políticas fiscal y monetaria, respectivamente. En este trabajo se sopesa la evidencia empírica sobre la existencia del ciclo político de tipo oportunista en la economía mexicana. Se toma en cuenta el trabajo de Nordhaus (1975), quien analiza el problema de la elección entre inflación y desempleo con la curva de Phillips con expectativas adaptativas para explicar que los ciclos económicos son provocados por el comportamiento oportunista del gobierno en turno. Sin embargo, en este trabajo se agrega otro enfoque, ya que las variables, la teoría económica y la metodología son distintas. En general, se realiza un análisis de regresión mediante dos métodos: mínimos cuadrados ordinarios y vectores autorregresivos. El primero busca identificar el efecto de cada una de las variables explicativas en el crecimiento económico medido por el PIB real en un entorno de estática comparativa y considerando que las variables explicativas son exógenas y tienen un impacto lineal. Por su parte, el segundo trata de encontrar la relación dinámica entre las variables y su interrelación al considerarlas todas endógenas, al tiempo que permite estudiar los impactos de una variable a partir de las funciones de impulso-respuesta. En 
ambos casos, las variables de estudio ${ }^{2}$ son el producto interno bruto (PIB), los egresos del sector público presupuestario denominados gasto público $(G P)$, la base monetaria $(B M)$ y la tasa de inflación (INF) para el periodo 1960-2011. La lógica de usar estas variables consiste en que la manera de llevar a cabo una política fiscal expansiva generalmente está asociada a un incremento del gasto público, mientras que la política monetaria está vinculada a movimientos de la base monetaria, como ha ocurrido en fechas recientes en el caso de Japón con la denominada "Abenomics", política económica instrumentada por el primer ministro Shinzo Abe para controlar la inflación e impulsar el crecimiento económico. Respecto a la razón para incorporar la tasa de inflación al modelo, es que si las políticas fiscal y monetaria están teniendo efectos en el crecimiento del PIB real, se deberían estar generando presiones inflacionarias, ya que se estaría cerrando la brecha del producto, situación que indicaría claramente que las acciones de política económica expansivas estarían impactando de manera positiva en el crecimiento económico. Las series son trimestrales y para las cuatro variables se utiliza la variación anual porcentual de los valores a precios de 2003. El PIB se estimó con los datos trimestrales de 1959 a 1979, con base en el coeficiente de estacionalidad que resulta de la misma serie con cifras trimestrales observadas a partir de 1980; dicho método se utilizó para la base monetaria en el periodo 1959-1985 con cifras observadas a partir del cuarto trimestre de 1985; lo mismo se hizo para los egresos del sector público presupuestario en el periodo 1959-2011, pero como de esta serie no se obtuvieron datos trimestrales y dado que su comportamiento anual es parecido al del gasto presupuestal del sector público, se decidió usar esta última serie observada a partir del tercer trimestre de 1977. Debido a que en México se tienen series estadísticas históricas a partir de 1960 sólo para escasas variables, no era viable incluir otras de menor temporalidad en el presente trabajo. Además, se consideran sólo los impactos de las elecciones presidenciales, que tienen lugar cada seis años, y de las legislativas (senadores y diputados federales) que coin-

${ }^{2}$ Las fuentes de información son: PIB, de 1959 a 1980, del Sistema de Cuentas Nacionales de México (SCNM), y de 1981 a 2011, del Banco de Información Económica (BIE), ambos del Instituto Nacional de Estadística y Geografía (INEGI). Base monetaria, de 1960 a 1983, del Anuario Estadístico de los Estados Unidos Mexicanos, del INEGi, y de 1983 a 2011, del Banco de México (Banxico). Tasa de inflación de 1959 a 1975, del Índice de precios al mayoreo de la Ciudad de México, del INEGI, y de 1976 a 2011, de Banxico. Gasto presupuestal del sector público, de 1959 a 2011, de Banxico. Egresos del sector público presupuestario, de 1959 a 1979, del del Anuario Estadístico de los Estados Unidos Mexicanos, y de 1980 a 2010, del Sistema de Finanzas Públicas, de la Secretaría de Hacienda y Crédito Público (SHCP). 
ciden con las primeras, omitiendo las intermedias, ya que se espera que su incidencia conjunta en la economía mexicana sea mayor. Para medir el efecto de los comicios se usa la variable cualitativa $E L E C$, que mide los trimestres anterior, simultáneo y posterior a la fecha de las elecciones y toma los valores de 1 en los tres últimos trimestres del año y de 0 en otro caso.

\section{Método de mínimos cuadrados ordinarios}

El modelo de regresión de mínimos cuadrados ordinarios sigue el método de interacciones entre variables cuantitativas y cualitativas, también conocido como modelos de análisis de covarianza (ANCOVA). ${ }^{3}$ Estos modelos tienen la siguiente forma general:

$$
Y=\alpha_{0}+\alpha_{1-n} X_{i-n}+\beta_{1-n} X_{i-n} D+\delta_{1-n} D+\varepsilon_{t},
$$

donde $Y$ es la variable dependiente; $\alpha_{0}$ es la ordenada al origen; $\alpha_{1-n}, \beta_{1-n}$ y $\delta_{1-n}$ son los parámetros de las variables cualitativas y cuantitativas; $X_{i}$ son las variables independientes; $D$ es la variable cualitativa y $\varepsilon_{t}$ es el término de error.

A cada modelo se le realizaron las pruebas estadísticas de linealidad, normalidad, no autocorrelación y no heterocedasticidad, para probar los supuestos sobre los errores estimados del método de mínimos cuadrados ordinarios (Gujarati 2010), esperando que sean MELI (mejor estimador lineal insesgado). También se recurre a la $R^{2}$, que indica si la(s) variable(s) independiente(s) explica(n) la evolución de la variable dependiente.

Para empezar, se analizan las series a lo largo del periodo de estudio (1960-2011) con la prueba de estabilidad de Chow; el resultado es que las cuatro series presentan un problema de cambio estructural entre los años 1982 y 1983. El cuadro A.1 del anexo estadístico muestra que el valor del estadístico $t$ es mayor a 0.05 para las cuatro variables, lo que indica que las series presentan un cambio estructural en 1982, hecho que puede explicarse por el sobreendeudamiento que comenzó a mostrar la economía mexicana a consecuencia del auge petrolero; por lo anterior, se decidió dividir en dos submuestras el periodo de estudio, 1960-1983 y 1984-2011, a fin de que el modelo estuviera mejor especifica-

\footnotetext{
${ }^{3}$ Son modelos basados en el análisis de varianza (ANOVA), pero que adicionalmente proporcionan un método que controla estadísticamente los efectos de las variables regresoras cuantitativas en modelos que también incluyen variables cualitativas (Gujarati, 2010, pp. 278 y 284).
} 
do y que los efectos de las variables explicativas no estuvieran contaminados por el cambio estructural. El modelo en ambos periodos tiene la siguiente forma general:

$$
\begin{aligned}
P I B=\beta_{0} & +\beta_{1} G P+\beta_{2} B M+\beta_{3} I N F+\beta_{4} P I B_{t-n}+\beta_{5} G P_{t-n}+\beta_{6} B M_{t-n}+\beta_{7} I N F_{t-n} \\
& +\beta_{8} P I B_{t-n} * E L E C+\beta_{9} G P_{t-n} * E L E C+\beta_{10} B M_{t-n} * E L E C \\
& +\beta_{11} I N F_{t-n} * E L E C
\end{aligned}
$$

donde $P I B, G P, B M$ e $I N F$ son las tasas de variación del PIB, el gasto público, la base monetaria y la inflación; $P I B_{t-n}, G P_{t-n}, B M_{t-n}$ e $I N F_{t-n}$ son las variables mencionadas rezagadas $n$ trimestres; $P I B_{t-n} * E L E C, G P_{t-n} * E L E C, B M_{t-n}$ e INF $F_{t-n}$ * ELEC representan la interacción entre factores cuantitativos y cualitativos que reflejan el comportamiento de las variables de estudio cuando suceden las elecciones; $\beta_{0}$ es la ordenada al origen, $\beta_{1,2, \ldots, n}$ son los parámetros de cada variable; $E L E C$ es la variable cualitativa; y $\varepsilon_{t}$ es el término estocástico. Las variables explicativas y el número de sus rezagos óptimos por modelo se determinará si son estadísticamente significativos de forma individual y considerando también los criterios de Akaike y de Schwarz.

\section{a) Estimaciones para el periodo $1960_{1}-1983_{4}$}

A continuación se modela el comportamiento de la variación del PIB ante cambios en las variables explicativas antes mencionadas para el periodo que va del primer trimestre de 1960 al cuarto de $1983\left(1960_{1}-1983_{4}\right)$. Los resultados se muestran en el cuadro 1.

Todas las variables explicativas son significativas con una probabilidad de 5\%, y el número de rezagos óptimo es de tres. Por un lado, los coeficientes indican que durante las elecciones, lo siguiente provoca un efecto negativo en la variación de $P I B$ : la interacción entre la variable que mide el efecto de las elecciones y las variables base monetaria, $B M * E L E C$, e inflación, $I N F * E L E C$, ambas al momento de los comicios; la inflación rezagada dos trimestres, $I N F(-2) * E L E C$; la base monetaria rezagada tres trimestres, $B M(-3) * E L E C$, y la inflación también rezagada tres trimestres, $\operatorname{INF}(-3) * E L E C$. Por otro lado, todas estas variables provocan efectos positivos en la variación de PIB: base monetaria rezagada un trimestre, $B M(-1)$, y tres trimestres, $B M(-3)$; inflación rezagada tres trimestres, $I N F(-3)$; la interacción entre la variable $E L E C$ y las va- 
Cuadro 1. Modelo de regresión para las elecciones presidenciales en México, $1960_{1}-1983_{4}$

Variable dependiente: PIB

Mínimos cuadrados ordinarios

Periodo (ajustado): 1961 $-1983_{4}$

Observaciones: 92 después de ajuste

Convergencia lograda después de 19 interacciones

\begin{tabular}{|c|c|c|c|c|}
\hline Variable & Coeficiente & Error estándar & Prueba $t$ & Probabilidad \\
\hline$C$ & 0.029278 & 0.005723 & 5.115410 & 0.0000 \\
\hline INF & -0.120886 & 0.024010 & -5.034905 & 0.0000 \\
\hline$B M(-1)$ & 0.190669 & 0.039890 & 4.779918 & 0.0000 \\
\hline$B M(-3)$ & 0.184753 & 0.050181 & 3.681756 & 0.0004 \\
\hline INF(-3) & 0.177554 & 0.032670 & 5.434732 & 0.0000 \\
\hline$B M^{*} E L E C$ & -12.873830 & 5.046500 & -2.551042 & 0.0128 \\
\hline$I N F^{*} E L E C$ & - 11.973390 & 4.683828 & -2.556326 & 0.0127 \\
\hline$B M(-1) * E L E C$ & 11.687000 & 4.537707 & 2.575531 & 0.0120 \\
\hline$I N F(-1) * E L E C$ & 15.088350 & 5.808945 & 2.597433 & 0.0114 \\
\hline$G P(-2) * E L E C$ & 0.150936 & 0.046298 & 3.260075 & 0.0017 \\
\hline$B M(-2) * E L E C$ & 0.688043 & 0.286734 & 2.399587 & 0.0190 \\
\hline$I N F(-2) * E L E C$ & -1.758165 & 0.669392 & -2.626511 & 0.0105 \\
\hline$B M(-3) * E L E C$ & -0.463416 & 0.198754 & -2.331609 & 0.0225 \\
\hline$I N F(-3) * E L E C$ & -0.730951 & 0.305011 & -2.396471 & 0.0191 \\
\hline$D 68$ & 0.031398 & 0.008999 & 3.488961 & 0.0008 \\
\hline$D 69$ & -0.034880 & 0.008961 & - 3.892598 & 0.0002 \\
\hline$D 72$ & 0.036230 & 0.012283 & 2.949682 & 0.0043 \\
\hline$D 80$ & 0.040120 & 0.007809 & 5.137895 & 0.0000 \\
\hline$A R(1)$ & 0.290410 & 0.123253 & 2.356215 & 0.0211 \\
\hline$R^{2}$ & 0.890074 & \multicolumn{2}{|c|}{$\begin{array}{l}\text { Significación de la variable } \\
\text { dependiente }\end{array}$} & 0.059315 \\
\hline$R^{2}$ ajustada & 0.862969 & \multicolumn{2}{|c|}{$\begin{array}{l}\text { Desviación estándar de la } \\
\text { variable dependiente }\end{array}$} & 0.033794 \\
\hline $\begin{array}{l}\text { Error estándar de la } \\
\text { regresión }\end{array}$ & 0.012510 & \multicolumn{2}{|c|}{$\begin{array}{l}\text { Criterio de información de } \\
\text { Akaike }\end{array}$} & -5.742893 \\
\hline $\begin{array}{l}\text { Suma de cuadrados de los } \\
\text { residuos }\end{array}$ & 0.011424 & \multicolumn{2}{|c|}{ Criterio de Schwarz } & -5.222089 \\
\hline Función de verosimilitud & 283.173100 & \multicolumn{2}{|l|}{ Prueba $F$} & 32.837940 \\
\hline Test de Durbin-Watson & 1.931979 & \multirow{2}{*}{\multicolumn{2}{|c|}{ Probabilidad (prueba $F$ ) }} & 0.000000 \\
\hline Raíces $A R$ invertidas & 0.29 & & & \\
\hline
\end{tabular}

Nota: Los subíndices indican trimestres del año respectivo.

Fuente: Elaboración propia con el programa Eviews 5. 
riables base monetaria, $B M(-1) * E L E C$, e inflación, $I N F(-1) * E L E C$, ambas rezagadas un trimestre, así como gasto público, $G P(-2) * E L E C$, y base monetaria, $B M(-2) * E L E C$, ambas rezagadas dos trimestres. La $R^{2}$ indica que las variables explican el comportamiento de la tasa de crecimiento de PIB en $86.3 \%$. Para corregir el modelo se hizo uso de cuatro variables cualitativas como son $D 68, D 69, D 72$ y $D 80$, además de un modelo autorregresivo de orden uno, $A R(1)$. Con ello, el modelo pasa las pruebas de evaluación de los supuestos que se presentan en el cuadro A.2 del anexo estadístico. En términos generales, se observa que el efecto de las elecciones medido con la variable ELEC sí influye en el comportamiento de $P I B$ antes, durante y después del trimestre de las elecciones, por lo que se acepta que para esta submuestra hay evidencia de que existe un ciclo político en la economía mexicana.

\section{b) Estimaciones para el periodo $1984_{1}-2011_{4}$}

En esta sección se analiza el comportamiento del PIB ante cambios en las variables explicativas durante el periodo del primer trimestre de 1984 al cuarto de $2011\left(1984_{1}-2011_{4}\right)$. Los resultados se muestran en el cuadro 2.

En dicho cuadro se aprecia que las variables gasto público, $G P$, e inflación, INF, al momento de las elecciones; inflación rezagada dos trimestres, $I N F(-2)$, y base monetaria rezagada tres trimestres, $B M(-3)$, influyen de manera negativa en la economía. Se aprecia que la inflación rezagada dos trimestres es la que más responde conforme varía el PIB. Por el contrario, las variables base monetaria, $B M(-1)$, e inflación, $I N F(-1)$, ambas rezagadas un trimestre, e inflación rezagada tres trimestres $I N F(-3)$, así como la interacción entre la base monetaria rezagada un trimestre y la variable cualitativa para medir el efecto de las elecciones, $B M(-1) * E L E C$, permiten un efecto acelerador en la economía, aunque poco significativo de acuerdo al valor de sus coeficientes. En este caso, los resultados indican que las elecciones influyen muy poco en el comportamiento de la economía, con excepción de la base monetaria, que sí sufre la influencia de los políticos en años electorales. La $R^{2}$ indica que las variables explican el comportamiento de la tasa de crecimiento de $P I B$ en $77.9 \%$. Además. se corrigió el modelo con tres variables cualitativas como son $D 93, D 95$ y $D 09$, y un $A R(1)$. Con ello, pasa las pruebas de evaluación de los supuestos que se presentan en el cuadro A.3 del anexo estadístico. En conclusión, respecto al caso anterior, es claro que el efecto de las elecciones medido con la variable ELEC no influye en el comportamiento de PIB antes, durante y después del trimestre de los comicios, 
por lo que no hay suficiente evidencia para suponer un ciclo político en la economía mexicana en esta submuestra.

Cuadro 2. Modelo de regresión para las elecciones presidenciales en México,

$$
1984_{1}-2011_{4}
$$

Variable dependiente: $P I B$

Mínimos cuadrados ordinarios

Periodo: $1984_{1}-2011_{4}$

Observaciones: 112

Convergencia lograda después de 9 interacciones

\begin{tabular}{|c|c|c|c|c|}
\hline Variable & Coeficiente & Error estándar & Prueba $t$ & Probabilidad \\
\hline C & 0.026605 & 0.007643 & 3.480906 & 0.0007 \\
\hline$G P$ & -0.045890 & 0.023487 & -1.953884 & 0.0535 \\
\hline INF & -0.069753 & 0.030025 & -2.323186 & 0.0222 \\
\hline$B M(-1)$ & 0.100832 & 0.028403 & 3.550035 & 0.0006 \\
\hline$I N F(-1)$ & 0.153327 & 0.048972 & 3.130882 & 0.0023 \\
\hline$I N F(-2)$ & -0.186851 & 0.046863 & - 3.987197 & 0.0001 \\
\hline$B M(-3)$ & -0.048620 & 0.025278 & -1.923381 & 0.0573 \\
\hline$I N F(-3)$ & 0.103348 & 0.029042 & 3.558643 & 0.0006 \\
\hline$B M(-1) * E L E C$ & 0.105372 & 0.041833 & 2.518902 & 0.0134 \\
\hline D93 & 0.050811 & 0.014907 & 3.408587 & 0.0009 \\
\hline D95 & -0.063199 & 0.015140 & -4.174330 & 0.0001 \\
\hline D09 & -0.065555 & 0.014318 & -4.578560 & 0.0000 \\
\hline$A R(1)$ & 0.693725 & 0.074237 & 9.344760 & 0.0000 \\
\hline$R^{2}$ & 0.778733 & \multicolumn{2}{|c|}{$\begin{array}{l}\text { Significación de la variable } \\
\text { dependiente }\end{array}$} & 0.026027 \\
\hline$R^{2}$ ajustada & 0.751912 & \multicolumn{2}{|c|}{$\begin{array}{l}\text { Desviación estándar de la } \\
\text { variable dependiente }\end{array}$} & 0.036218 \\
\hline $\begin{array}{l}\text { Error estándar de la } \\
\text { regresión }\end{array}$ & 0.018040 & \multicolumn{2}{|c|}{$\begin{array}{l}\text { Criterio de información de } \\
\text { Akaike }\end{array}$} & -5.083735 \\
\hline $\begin{array}{l}\text { Suma de cuadrados de } \\
\text { los residuos }\end{array}$ & 0.032217 & \multicolumn{2}{|c|}{ Criterio de Schwarz } & -4.768194 \\
\hline Función de verosimilitud & 297.689100 & \multicolumn{2}{|l|}{ Prueba $F$} & 29.035210 \\
\hline Test de Durbin-Watson & 2.013743 & \multirow{2}{*}{\multicolumn{2}{|c|}{ Probabilidad (prueba $t$ ) }} & 0.000000 \\
\hline Raíces $A R$ invertidas & 0.69 & & & \\
\hline
\end{tabular}

Fuente: Elaboración propia con el programa Eviews 5. 


\section{Vectores autorregresivos}

En este apartado se ocupan las mismas variables y frecuencias de tiempo definidas con el método de mínimos cuadrados ordinarios, pero ahora se supone que todas las variables son endógenas y que existe una interacción dinámica entre ellas, al tiempo que se identifica el impacto estocástico en dichas variables a través de la estimación de las funciones de impulso-respuesta y la descomposición de la varianza. A los modelos de vectores autorregresivos estimados se les realizaron las pruebas de autocorrelación, normalidad y heterocedasticidad para que los resultados tengan un nivel de robustez importante. Además, se realizó la prueba de causalidad de Granger-Sims entre las variables endógenas para ver si hay una relación causal entre ellas en el largo plazo. Los modelos de vectores autorregresivos a utilizar para cada periodo tienen la siguiente forma general:

$$
\begin{gathered}
P I B=a_{0}+a_{1-n} P I B_{t-1-n}+b_{2-n} G P_{t-1-n}+c_{3-n} B M_{t-1-n}+d_{4-n} I N F_{t-1-n}+e_{1} \\
G P=b_{0}+b_{1-n} G P_{t-1-n}+c_{2-n} B M_{t-1-n}+d_{3-n} I N F_{t-1-n}+a_{4-n} P I B_{t-1-n}+e_{2} \\
B M=c_{0}+c_{1-n} B M_{t-1-n}+d_{2-n} I N F_{t-1-n}+a_{3-n} P I B_{t-1-n}+b_{4-n} G P_{t-1-n}+e_{3} \\
I N F=d_{0}+d_{1-n} I N F_{t-1-n}+a_{2-n} P I B_{t-1-n}+b_{3-n} G P_{t-1-n}+c_{4-n} B M_{t-1-n}+e_{4},
\end{gathered}
$$

donde $a, b, c y d$ son los coeficientes del intercepto y de las variables rezagadas $n$ veces, y $e_{1,2,3 \text { y } 4}$ son los errores de cada ecuación. Al igual que con los mínimos cuadrados ordinarios, se contempla la variable cualitativa ELEC para constatar el efecto de las elecciones. A continuación se exhiben los resultados para la primer submuestra.

\section{a) Vectores autorregresivos. Periodo $1960_{1}-1983_{4}$}

En este modelo, el número de rezagos óptimos es de seis e incluye ELEC como variable exógena. Una vez que el modelo pasó las pruebas de evaluación de los supuestos presentes en el cuadro A.4 del anexo estadístico, se procedió a realiza la prueba de Granger para identificar si hay causalidad entre las variables de estudio.

El criterio de evaluación es que la probabilidad debe ser menor a 0.05 para aceptar la hipótesis de causalidad. En el cuadro 3 se aprecia que ninguna otra variable causa $P I B$, mientras que $I N F$, sí causa a $B M$. Asimismo, $G P$ sí es cau- 
sada por la variable $I N F$, al igual que por $P I B$, aunque en este último caso a un nivel de significación de $5.1 \%$. Por último, $I N F$ es causada por las variables $P I B$ y $B M$. A continuación, se hace el análisis de las gráficas de impulso-respuesta para apreciar la respuesta de $P I B$ ante cambios en el gasto público, la base monetaria y la inflación.

Cuadro 3. Vectores autorregresivos: $1960,-1983_{4}$. Prueba de causalidad de Granger

Periodo: $1960_{1}-1983_{4}$

Observaciones: 90

\begin{tabular}{|l|c|c|c|}
\hline \multicolumn{4}{|l|}{ Variable dependiente: $P I B$} \\
\hline Excluye & $X^{2}$ & $\begin{array}{c}\text { Grados de } \\
\text { libertad }\end{array}$ & Probabilidad \\
\hline$G P$ & 0.930255 & 6 & 0.9881 \\
\hline$B M$ & 4.786033 & 6 & 0.5715 \\
\hline$I N F$ & 10.34481 & 6 & 0.1109 \\
\hline Todas & 21.31487 & 18 & 0.2638 \\
\hline
\end{tabular}

\begin{tabular}{|l|c|c|c|}
\hline \multicolumn{4}{|l|}{ Variable dependiente: $B M$} \\
\hline Excluye & $X^{2}$ & $\begin{array}{c}\text { Grados de } \\
\text { libertad }\end{array}$ & Probabilidad \\
\hline$P I B$ & 7.025797 & 6 & 0.3185 \\
\hline$G P$ & 3.693977 & 6 & 0.7180 \\
\hline$I N F$ & 46.30361 & 6 & 0.0000 \\
\hline Todas & 71.33734 & 18 & 0.0000 \\
\hline
\end{tabular}

\begin{tabular}{|l|c|c|r|}
\hline \multicolumn{4}{|l|}{ Variable dependiente: GP } \\
\hline Excluye & $X^{2}$ & $\begin{array}{c}\text { Grados de } \\
\text { libertad }\end{array}$ & Probabilidad \\
\hline$P I B$ & 12.52294 & 6 & 0.0513 \\
\hline$B M$ & 8.394244 & 6 & 0.2106 \\
\hline$I N F$ & 23.25259 & 6 & 0.0007 \\
\hline Todas & 48.5704 & 18 & 0.0001 \\
\hline
\end{tabular}

\begin{tabular}{|l|c|c|c|}
\hline \multicolumn{4}{|l|}{ Variable dependiente: INF } \\
\hline Excluye & $X^{2}$ & $\begin{array}{c}\text { Grados de } \\
\text { libertad }\end{array}$ & Probabilidad \\
\hline$P I B$ & 32.00333 & 6 & 0.0000 \\
\hline$G P$ & 1.151648 & 6 & 0.9792 \\
\hline$B M$ & 58.44929 & 6 & 0.0000 \\
\hline Todas & 92.53701 & 18 & 0.0000 \\
\hline
\end{tabular}

Fuente: Elaboración propia con el programa Eviews 5.

Gráfica 1. Vectores autorregresivos: 1960,-19834. Función impulso-respuesta

a) Respuesta de $P I B$ a GP

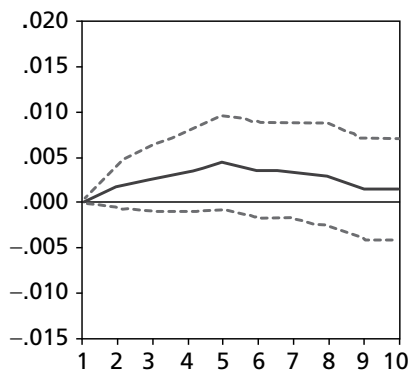

b) Respuesta de $P I B$ a $B M$

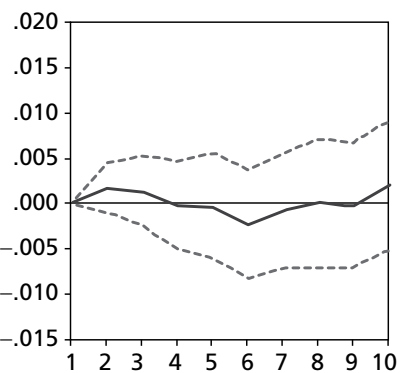

c) Respuesta de PIB a INF

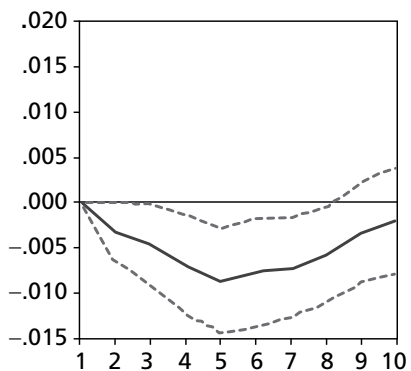

Fuente: Elaboración propia con el programa Eviews 5. 
La gráfica 1.a muestra que la respuesta de $P I B$ ante un cambio en el gasto público es positiva hasta el quinto trimestre, a partir del cual la actividad económica empieza a decrecer. A su vez, la gráfica 1.b muestra que PIB también responde positivamente ante cambios de la base monetaria hasta el trimestre dos; después empieza decrecer hasta el noveno trimestre, cuando empieza a recuperarse. En contraste, la gráfica 1.c ilustra que la respuesta de $P I B$ ante variaciones en la inflación es negativa, ya que a medida que aumenta el nivel de precios la actividad económica disminuye, principalmente desde el primer trimestre, aunque luego del quinto vuelve a crecer ligeramente.

Los vectores para la primera submuestra (véase cuadro A.6 del anexo) no evidencian efectos dinámicos en el tiempo entre las variables, aunque sí relaciones entre algunas variables y sus rezagos. Entre las más significativas están: el PIB con sus rezagos del primer y cuarto trimestre; el gasto público con sus rezagos del primero, el cuarto y el quinto trimestre; la base monetaria y la inflación con los rezagos de la base monetaria del primer y quinto trimestre; el PIB, la base monetaria y la inflación con ésta rezagada un trimestre. Al respecto, la variable ELEC sólo es significativa, aunque sea poco, con la inflación. Lo anterior motivó la revisión de la descomposición de la varianza de $P I B$.

El cuadro 4 muestra los resultados de la descomposición de la varianza de $P I B$ en distintos periodos de tiempo ante impactos de diferentes variables. Se observa que la varianza de $P I B$ se explica a sí misma en gran medida a corto plazo con variaciones superiores a $90 \%$ y va disminuyendo en el largo plazo hasta llegar a $58 \%$. Además, a largo plazo su varianza se explica en $6.4 \%$ y $1.2 \%$ por el gasto público y la base monetaria, respectivamente, y aunque ésta permaneció en esos niveles de variación, el gasto público explica la varianza de $P I B$ en $8.1 \%$ en el largo plazo, es decir, que los impactos en el gasto público a partir del mediano plazo explican considerablemente esta varianza. Lo anterior se aprecia en la inflación, la cual responde en gran medida al impacto económico desde el corto plazo con una variación de 6.7\%, llegando en el largo plazo incluso a 32\%.

En términos generales, los resultados de este modelo para el periodo $1960_{1}-1983_{4}$ indican que las variables de estudio gasto público, base monetaria e inflación no explican en conjunto la variación del PIB, siendo que sólo la inflación deja sentir su influencia en la economía, principalmente en el largo plazo, aunque no en la magnitud esperada. Por lo tanto, para este modelo no se acepta la hipótesis de la presencia de un ciclo político en la economía mexicana en la submuestra estudiada. A continuación se analiza la otra parte del periodo, es decir, de $1984_{1}$ a $2011_{4}$. 
212 ECONOMÍA: TEORÍA Y PRÁCTICA • Nueva Época, número 40, enero-junio 2014

Cuadro 4. Vectores autorregresivos: $1960_{1}-1983_{4}$. Descomposición de la varianza

\begin{tabular}{|l|c|c|c|c|c|}
\hline Periodo & Error estándar & PIB & GP & $B M$ & INF \\
\hline 1 & 0.014292 & 100.000000 & 0.000000 & 0.000000 & 0.000000 \\
\hline 2 & 0.019186 & 95.391910 & 0.915514 & 0.728109 & 2.964466 \\
\hline 3 & 0.022300 & 90.397620 & 2.051857 & 0.884641 & 6.665885 \\
\hline 4 & 0.025367 & 83.344310 & 3.258455 & 0.689401 & 12.707840 \\
\hline 5 & 0.027310 & 72.859170 & 5.422158 & 0.609397 & 21.109270 \\
\hline 6 & 0.028776 & 66.242220 & 6.429025 & 1.162521 & 26.166230 \\
\hline 7 & 0.029877 & 61.567660 & 7.309045 & 1.149763 & 29.973540 \\
\hline 8 & 0.030581 & 58.880860 & 7.912966 & 1.098282 & 32.107890 \\
\hline 9 & 0.030812 & 58.102360 & 8.039748 & 1.085310 & 32.772580 \\
\hline 10 & 0.031136 & 57.942000 & 8.104646 & 1.488298 & 32.465060 \\
\hline
\end{tabular}

Fuente: Elaboración propia con el programa Eviews 5.

\section{b) Vectores autorregresivos. Periodo $1984_{1}^{-2011_{4}}$}

Como en el caso anterior, el número de rezagos óptimos es seis y los resultados aprobatorios de las pruebas de evaluación de los supuestos se consignan en el cuadro A.5 del anexo. Por su parte, la prueba de causalidad de Granger presentó los resultados que se muestran en el cuadro 5 .

Cuadro 5. Vectores autorregresivos: $1984_{1}-2011_{4}$. Prueba de causalidad de Granger Periodo: $1984_{1}-2011_{4}$

Observaciones: 112

\begin{tabular}{|l|c|c|c|}
\hline \multicolumn{4}{|l|}{ Variable dependiente: PIB } \\
\hline Excluye & $X^{2}$ & $\begin{array}{c}\text { Grados de } \\
\text { libertad }\end{array}$ & Probabilidad \\
\hline$G P$ & 3.252357 & 6 & 0.7766 \\
\hline$B M$ & 9.727067 & 6 & 0.1366 \\
\hline$I N F$ & 7.208291 & 6 & 0.302 \\
\hline Todas & 18.38523 & 18 & 0.4306 \\
\hline
\end{tabular}

\begin{tabular}{|l|c|c|c|}
\hline \multicolumn{4}{|l|}{ Variable dependiente: $B M$} \\
\hline Excluye & $X^{2}$ & $\begin{array}{c}\text { Grados de } \\
\text { libertad }\end{array}$ & Probabilidad \\
\hline$P I B$ & 4.685467 & 6 & 0.5847 \\
\hline$G P$ & 11.11254 & 6 & 0.0850 \\
\hline$I N F$ & 19.09588 & 6 & 0.0040 \\
\hline Todas & 35.23377 & 18 & 0.0088 \\
\hline
\end{tabular}

\begin{tabular}{|l|c|c|c|}
\hline \multicolumn{4}{|l|}{ Variable dependiente: $G P$} \\
\hline Excluye & $X^{2}$ & $\begin{array}{c}\text { Grados de } \\
\text { libertad }\end{array}$ & Probabilidad \\
\hline$P I B$ & 2.878099 & 6 & 0.824 \\
\hline$B M$ & 8.120633 & 6 & 0.2294 \\
\hline$I N F$ & 20.49471 & 6 & 0.0023 \\
\hline Todas & 34.14495 & 18 & 0.0121 \\
\hline
\end{tabular}

\begin{tabular}{|l|c|c|c|}
\hline \multicolumn{4}{|l|}{ Variable dependiente: INF } \\
\hline Excluye & $X^{2}$ & $\begin{array}{c}\text { Grados de } \\
\text { libertad }\end{array}$ & Probabilidad \\
\hline$P I B$ & 5.429217 & 6 & 0.4901 \\
\hline$G P$ & 20.02872 & 6 & 0.0027 \\
\hline$B M$ & 2.239015 & 6 & 0.8965 \\
\hline Todas & 33.99392 & 18 & 0.0126 \\
\hline
\end{tabular}

Fuente: Elaboración propia con el programa Eviews 5. 
Como se aprecia, ninguna variable causa a $P I B$, mientras que $B M$ sí es causada por $I N F$ e incluso por $G P$, al menos, en este último caso, con una probabilidad de $8.5 \%$. Por otra parte, $I N F$ sí es causa de $G P$ y, a su vez, $G P$ es causa de $I N F$. A continuación, se realiza al análisis de las gráficas de impulso-respuesta para apareciar la respuesta de PIB ante cambios en el gasto público, la base monetaria y la inflación.

Gráfica 2. Vectores autorregresivos: $1984_{1}-2011_{4}$. Función impulso-respuesta

a) Respuesta de $P I B$ a GP

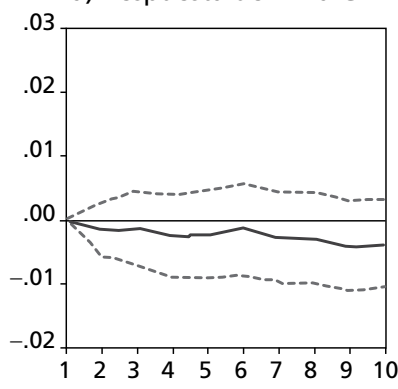

b) Respuesta de $P I B$ a $B M$

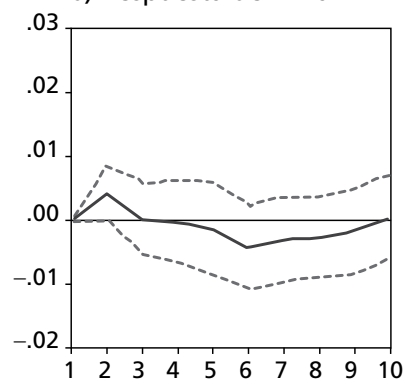

c) Respuesta de PIB a INF

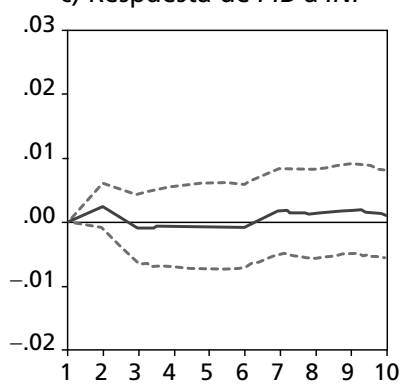

Fuente: Elaboración propia con el programa Eviews 5.

La gráfica 2.a muestra que desde el primer trimestre la incidencia del gasto público en $P I B$ es negativa. En la gráfica $2 . b$ se exhibe que la respuesta de $P I B$ es positiva ante cambios en la base monetaria hasta el segundo trimestre, luego, a partir del tercero, el impacto de ésta es negativo y en el décimo trimestre al parecer vuelve a incidir positivamente. En la gráfica 2.c es posible observar que la inflación mantiene estable el comportamiento de PIB a largo plazo. Hay que recordar que a partir de la década de los ochenta se empezaron a aplicar políticas de estabilización para mantener el nivel de precios, hecho que se nota en la respuesta de $P I B$ ante incrementos en la inflación; si bien entre los trimestres dos y seis, a medida que crece la inflación, $P I B$ decrece, a partir del trimestre siete esto cambia ligeramente. Los vectores para la segunda submuestra (véase cuadro A.7 del anexo) tampoco muestran efectos dinámicos en el tiempo entre las variables, pero al igual que en la primer submuestra sí se observan relaciones. Las más relevantes son: el PIB y su rezago del primer trimestre; el gasto público y la inflación con el gasto público rezagado un trimestre, y el gasto público con su rezago del cuarto trimestre. En el caso de los rezagos de la inflación, el más significativo es el del primer trimestre. Por su parte, la variable ELEC es estadísticamente significativa sólo con el gasto público y la inflación. 
214 ECONOMÍA: TEORÍA Y PRÁCTICA • Nueva Época, número 40, enero-junio 2014

Cuadro 6. Vectores autorregresivos: $1984_{1}-2011_{4}$. Descomposición de la varianza

\begin{tabular}{|c|c|c|c|c|c|}
\hline Periodo & Error estándar & $P I B$ & $G P$ & $B M$ & $I N F$ \\
\hline 1 & 0.021403 & 100.000000 & 0.000000 & 0.000000 & 0.000000 \\
\hline 2 & 0.029178 & 96.874240 & 0.305006 & 2.161724 & 0.659030 \\
\hline 3 & 0.034217 & 97.470680 & 0.382460 & 1.573570 & 0.573292 \\
\hline 4 & 0.035739 & 97.097870 & 0.872720 & 1.451964 & 0.577443 \\
\hline 5 & 0.035899 & 96.465290 & 1.283584 & 1.639669 & 0.611459 \\
\hline 6 & 0.036264 & 94.896300 & 1.413326 & 3.054032 & 0.636344 \\
\hline 7 & 0.036986 & 93.631320 & 1.951392 & 3.602984 & 0.814307 \\
\hline 8 & 0.037348 & 92.487870 & 2.502428 & 4.100096 & 0.909604 \\
\hline 9 & 0.037689 & 90.876860 & 3.698574 & 4.259040 & 1.165530 \\
\hline 10 & 0.037895 & 89.964070 & 4.575454 & 4.225145 & 1.235330 \\
\hline
\end{tabular}

Fuente: Elaboración propia con el programa Eviews 5.

En el cuadro 6 se aprecia que, tanto a corto como a largo plazo, PIB se explica en gran parte por sí misma, principalmente en el corto plazo, con $100 \%$, y registra la menor variación, de $90 \%$, en el largo plazo. De igual manera que en el análisis de la primera submuestra del estudio, conforme pasa el tiempo, el gasto público y la base monetaria explican la variación de $P I B$. En el mediano plazo, la base monetaria explica la variación de $P I B$ en $3 \%$, mientras que en el largo plazo llega a ser de $4.2 \%$. Por su parte, no es sino en el largo plazo que el gasto público explica la variación de $P I B$ en $4.6 \%$. Los impactos de la base monetaria explican en un mayor lapso la varianza de $P I B$, aunque los impactos en el gasto público y en la base monetaria no se reflejan en la inflación, ya que ésta registra variaciones poco relevantes, inferiores al $1 \%$, en el corto y mediano plazo, mientras que en el largo explica la variación de PIB en $1.2 \%$. Con lo anterior, se concluye que no hay suficiente evidencia para aceptar la hipótesis de la presencia de un ciclo político en la economía mexicana en el periodo $1984_{: 1}-2011_{: 4}$ con el método de vectores autorregresivos.

\section{El CICLO POLÍtico EN MÉXICO}

Los modelos anteriores muestran evidencia de un ciclo político para la submuestra de 1960 a 1983. En contraste, en la que comprende de 1984 a 2011, los resultados son débiles en el caso del método de vectores autorregresivos y la descomposición de la varianza, mientras que en el de mínimos cuadrados ordinarios los resultados no apoyan la hipótesis de la presencia de un ciclo político para el periodo estudiado. Lo anterior permite inferir que aunque en México está presente 
un ciclo político, sus impactos recientes se han limitado debido a la autonomía que tiene el Banco de México (central), que restringe parcialmente la aplicación de una política monetaria expansiva. Al mismo tiempo, existe una apertura comercial que puede generar filtraciones en el crecimiento y hace más difícil influir sobre el ciclo económico que en una economía cerrada. También la alternancia en el poder ha vuelto más complicado expandir el gasto, ya que se tiene mejor rendición de cuentas, auditoría de los recursos públicos y mayor transparencia de las administraciones. Se presume que los efectos sobre el total de la economía han disminuido por los elementos referidos, aunque el gasto y el endeudamiento de algunos estados y municipios se han incrementado de manera importante. Ejemplos como el de Coahuila son ilustrativos en fechas recientes, por lo que seguramente el ciclo político pasó de un esquema federal a uno focalizado en lo local. Esta situación ha llevado a promover una iniciativa de ley para limitar el endeudamiento de los estados y que haya mayor transparencia.

\section{El ciclo político se debilita}

Los resultados del modelo indican que de 1960 a 1983 hay evidencia de un ciclo político tradicional en la economía mexicana, mientras que de 1984 a 2011 se difumina su presencia. Se puede suponer que las políticas de estabilización implementadas en la década de los ochenta redujeron los efectos negativos provocados por los políticos en tiempo de elecciones. Además, los resultados corroboran al menos un supuesto de Nordhaus (1975) respecto a que los electores son miopes, pues sólo recuerdan los hechos más próximos a las elecciones y se basan en sus experiencias pasadas para emitir su voto; al menos así sucedió hasta que en el año 2000 fue electo presidente un candidato de un partido distinto al que había ocupado por décadas el poder. Las crisis de 1982, 1987 y 1994 fueron factores determinantes para la alternancia. Lo anterior confirma que la sociedad mexicana pone mayor atención a la situación de la economía nacional y sigue los pasos de los principales actores políticos que rigen la vida económica de México, es decir, el ciclo oportunista pierde sus efectos a partir de 1984 .

Si bien se esperaba que la evidencia fuera más notoria al considerar las elecciones federales y los datos a nivel nacional de las variables de estudio, los resultados proponen que los efectos serían más significativos si se consideran datos de un ámbito más restringido, como el estatal o el municipal, siempre que se tenga disponible la información respectiva. 
Aunque los efectos disminuyen en la segunda parte del periodo de estudio es claro que aún se aprecia la incidencia de los políticos en algunas variables económicas clave, como la base monetaria y la inflación. Lo anterior se explica en el sentido de que cuando la gente tiene más dinero a la mano y puede disponer de él a su gusto aumenta sus compras y su bienestar social, pero a la vez incrementa el nivel general de precios, así que al pasar las votaciones lo único que queda de esta fiesta electoral son altos niveles de inflación y, en consecuencia, el gobierno en turno aplica políticas de estabilidad para tratar de minimizar esos efectos negativos en la economía mexicana. Es notorio que la influencia de los políticos sobre la base monetaria disminuyó, con la autonomía del banco central, a partir de 1994, pero los resultados indican que no desapareció del todo. Para reducir los efectos negativos de la acción de los políticos en la economía, se presentan algunas propuestas que se describen a continuación.

\section{Propuestas constitucionales y de política económica}

Con base en los resultados econométricos, se proponen medidas que disminuyan el ciclo político, de tal manera que no altere la evolución económica. Los datos arrojados por los modelos realizados con mínimos cuadrados ordinarios y con vectores autorregresivos indican que el uso del gasto público como instrumento de los políticos para manipular la economía se dio principalmente en el periodo 1960-1983, cuando el gasto se incrementaba considerablemente en los años electorales. Se propone erradicar esta acción deliberada de los políticos con dos alternativas: la primera es limitar al Instituto Federal Electoral (IFE) como fuente de recursos casi inagotable para los partidos y la segunda es promover la reelección de los políticos como premio por su desempeño en el cargo. Para la primera opción se sugiere reformar el artículo 41 de la Constitución Política de los Estados Unidos Mexicanos (1917) respecto al monto y origen de los recursos, en específico se propone la reforma del primer párrafo de la fracción II, así como los incisos a) y b), y anexar un párrafo después del inciso c), como se detalla en el cuadro 7.

La propuesta tiene la finalidad de reducir el negocio para todos aquellos que se dedican a vivir de la política y no cumplen cabalmente con su trabajo, ya que no se les exigen resultados. Como incentivo para que los políticos tengan una buena gestión, básicamente se propone limitar los recursos a los partidos para que sólo a quienes les interese servir correctamente a la sociedad 
Cuadro 7. Propuesta de reforma al artículo 41 de la Constitución Política de México

\begin{tabular}{|c|c|}
\hline Vigente & Propuesta \\
\hline $\begin{array}{l}\text { II. La ley garantizará que los partidos políti- } \\
\text { cos nacionales cuenten de manera equita- } \\
\text { tiva con elementos para llevar a cabo sus } \\
\text { actividades y señalará las reglas a que se } \\
\text { sujetará el financiamiento de los propios } \\
\text { partidos y sus campañas electorales, de- } \\
\text { biendo garantizar que los recursos públicos } \\
\text { prevalezcan sobre los de origen privado. }\end{array}$ & $\begin{array}{l}\text { II. La ley garantizará que los partidos políticos } \\
\text { nacionales cuenten de manera equitativa con } \\
\text { elementos para llevar a cabo sus actividades } \\
\text { y señalará las reglas a que se sujetará el finan- } \\
\text { ciamiento de los propios partidos y sus cam- } \\
\text { pañas electorales, debiendo garantizar que se } \\
\text { especifique el origen y la aplicación de los recur- } \\
\text { sos públicos y privados. }\end{array}$ \\
\hline $\begin{array}{l}\text { a) El financiamiento público para el sos- } \\
\text { tenimiento de sus actividades ordinarias } \\
\text { permanentes se fijará anualmente, multi- } \\
\text { plicando el número total de ciudadanos } \\
\text { inscritos en el padrón electoral por el } 65 \% \\
\text { del salario mínimo diario vigente para } \\
\text { el Distrito Federal. El } 30 \% \text { de la cantidad } \\
\text { que resulte de acuerdo a lo señalado an- } \\
\text { teriormente, se distribuirá entre los parti- } \\
\text { dos políticos en forma igualitaria y el } 70 \% \\
\text { restante de acuerdo con el porcentaje de } \\
\text { votos que hubieren obtenido en la elección } \\
\text { de diputados inmediata anterior. }\end{array}$ & $\begin{array}{l}\text { a) El financiamiento para el sostenimiento de } \\
\text { sus actividades ordinarias permanentes no } \\
\text { debe ser mayor al } 0.05 \% \text { respecto al produc- } \\
\text { to interno bruto a precios constantes del año } \\
\text { anterior. Del monto total, el } 80 \% \text { del finan- } \\
\text { ciamiento será de carácter público y el } \mathbf{2 0 \%} \\
\text { restante será de carácter privado, y se fijará } \\
\text { anualmente, multiplicando el número total de } \\
\text { ciudadanos inscritos en el padrón electoral por } \\
\text { el } 65 \% \text { del salario mínimo diario vigente para } \\
\text { el Distrito Federal. El } 30 \% \text { de la cantidad que } \\
\text { resulte de acuerdo a lo señalado anteriormente } \\
\text { se distribuirá entre los partidos políticos en for- } \\
\text { ma igualitaria y el } 70 \% \text { restante de acuerdo con } \\
\text { el porcentaje de votos que hubieren obtenido } \\
\text { en la elección de diputados inmediata anterior. }\end{array}$ \\
\hline \multirow[t]{2}{*}{$\begin{array}{l}\text { b) El financiamiento público para las activi- } \\
\text { dades tendientes a la obtención del voto } \\
\text { durante el año en que se elijan presidente } \\
\text { de la República, senadores y diputados } \\
\text { federales, equivaldrá al } 50 \% \text { del financia- } \\
\text { miento público que le corresponda a cada } \\
\text { partido político por actividades ordinarias } \\
\text { en ese mismo año; cuando sólo se elijan } \\
\text { diputados federales, equivaldrá al } 30 \% \\
\text { de dicho financiamiento por actividades } \\
\text { ordinarias. }\end{array}$} & $\begin{array}{l}\text { b) El financiamiento para las actividades ten- } \\
\text { dientes a la obtención del voto durante el año } \\
\text { en que se elijan presidente de la República, } \\
\text { senadores y diputados federales, equivaldrá } \\
\text { al } 50 \% \text { del financiamiento total que le corres- } \\
\text { ponda a cada partido político por actividades } \\
\text { ordinarias en ese mismo año, y del total, el } 70 \% \\
\text { será financiado por recursos públicos y el } 30 \% \\
\text { restante por recursos privados. Cuando sólo se } \\
\text { elijan diputados federales, equivaldrá al } 30 \% \\
\text { de dicho financiamiento por actividades ordi- } \\
\text { narias, y del total, el } 80 \% \text { del financiamiento } \\
\text { será de carácter público y el } 20 \% \text { restante será } \\
\text { de carácter privado. }\end{array}$ \\
\hline & $\begin{array}{l}\text { Los } 31 \text { estados de la Federación y el Distrito } \\
\text { Federal se apegarán a lo establecido en la } \\
\text { fracción II y sus correspondientes incisos de } \\
\text { tal manera que sea un criterio generalmente } \\
\text { aceptado para la elección de cargos públicos de } \\
\text { los tres niveles de gobierno. }\end{array}$ \\
\hline
\end{tabular}


desarrollen su carrera política y poner una barrera a los que sólo busquen beneficios personales.

El límite de medio punto porcentual al financiamiento total a los partidos políticos resulta del análisis de los recursos destinados a éstos y a las elecciones de 2006. En ese año, el gasto para este rubro, según el Centro de Estudios de las Finanzas Públicas (2006), fue de MXN 4798 millones corrientes de acuerdo a la actividad prioritaria $\mathrm{R} 006$ denominada "Administrar las prerrogativas electorales y fiscalizar el gasto de los partidos y agrupaciones políticas" del Ramo Autónomo 22 Instituto Federal Electoral, del Presupuesto de Egresos de la Federación, lo que representó el $0.62 \%$ del PIB real de 2006.

Por esta razón, se propone que el límite del financiamiento total de los partidos para sus actividades ordinarias sea de $0.5 \%$ anual. Una tasa fija del PIB implica que si quieren más recursos tendrán que facilitar el crecimiento de la economía cada año. Y, por último, al establecer que el financiamiento de los partidos sea tanto público como privado, se obliga a los políticos a comprometerse con la sociedad de tal manera que ambas partes se beneficien mutuamente, es decir, que además de pedir el apoyo a través del voto también soliciten patrocinio económico, lo que los compromete con sus seguidores y, si desean seguir manteniendo ese respaldo y recibirlo de más personas, los obliga a realizar un buen trabajo durante su gestión.

Al eliminar de la norma que el financiamiento de los partidos políticos sea exclusivamente con recursos públicos, también se podría controlar el origen tanto de éstos como de los privados especificando su fuente mediante recibos de los partidos a los contribuyentes, los cuales, sólo para el caso de las aportaciones particulares, sean deducibles de impuestos como un premio a la confianza de la sociedad hacia sus representantes. Aunado a lo anterior, sería necesario que los partidos políticos especifiquen a detalle el destino final de los recursos, la fecha de aportación, su uso y el monto, para cumplir con los límites de gastos establecidos en el artículo 41 de la Constitución.

La segunda propuesta es la reelección como premio por un trabajo bien hecho. Si la sociedad considera que los políticos en funciones cumplieron con sus propuestas de campaña y que los resultados son visibles, entonces podrán votar por ellos para reelegirse sólo un periodo más. Para ello, se deben reformar los artículos 59, 83 y 94 de la Constitución Política de México, que hacen mención a los derechos y obligaciones de quienes ocupan los cargos de presidente de la República, diputados, senadores y magistrados; además del artículo 116, que se refiere a todos los cargos públicos. 
Por otra parte, los resultados de este estudio indican que la base monetaria tiende a responder positivamente a la influencia de las elecciones, principalmente en la primera submuestra. En el lapso que abarca ésta, después de la crisis de 1982, se tenían tasas de inflación superiores a 100\%, problema que en 1994 motivó al gobierno federal a otorgar la autonomía al Banco de México para que tuviera el control exclusivo de la política monetaria, eliminando la cesión de crédito interno neto al gobierno. En este tenor, la recomendación es seguir manteniendo la autonomía del banco central, así como su objetivo prioritario de preservar la estabilidad del poder adquisitivo de la moneda nacional, conforme se establece en el sexto párrafo del artículo 28 de la Constitución Política de los Estados Unidos Mexicanos (1917).

El análisis de los efectos del ciclo político también se puede extender al manejo del tipo de cambio, ya que éste es particularmente sensible a la influencia de factores políticos. Al respecto, México ha padecido severas devaluaciones, por lo que se recomienda que en la Comisión de Cambios el voto de calidad sea transferido a los funcionarios del Banco de México, para evitar cualquier tentación de volver a manipular la paridad cambiaria.

\section{CONCLUSIONES Y RECOMENDACIONES}

Los resultados en todo el periodo de estudio (1960-2011), mediante los métodos de mínimos cuadrados ordinarios y de vectores autorregresivos, demuestran la existencia parcial de un ciclo político. Durante la primera parte del periodo (1960-1983), la mayor influencia de las elecciones se registró en las variables de estudio PIB, base monetaria, inflación y gasto público, mientras que de 1984 en adelante los efectos disminuyen. Esto, por dos razones: una es que se dio prioridad a las políticas de estabilización implementadas en la década de los ochenta y la otra es que la miopía de los votantes mexicanos se supera a partir de las elecciones de 2000, cuando fue electo presidente Vicente Fox, candidato del Partido Acción Nacional. Las crisis de 1982, 1987 y 1994 fueron factores determinantes para el cambio del partido en el poder.

Otra consecuencia de los resultados es que los efectos de este ciclo político serían más significativos si se consideraran datos de nivel local y no nacional, como son los de carácter estatal o municipal, además de que se incrementaran las variables de análisis relacionadas con las políticas fiscal y monetaria: los impuestos, los subsidios y el tipo de cambio, entre otros. De igual manera, una mayor frecuencia en los datos, por ejemplo, mensual, daría mejores resultados que 
con los intervalos trimestrales que se usaron en ésta y en otras investigaciones. Por supuesto, siempre que se tenga información disponible para ciertas variables económicas a nivel local y para un periodo de estudio tan largo.

En el caso del análisis de los vectores autorregresivos, se observó que los primeros rezagos de las variables fueron los más significativos estadísticamente, lo que también hace suponer que la información mensual reportaría resultados más precisos que los obtenidos. En el segundo lapso de análisis (1984-2011) los efectos disminuyen, pero se aprecia la incidencia de los políticos en algunas variables económicas clave como la base monetaria y la inflación, aunque en menor medida gracias a que se otorgó autonomía al banco central a partir de 1994. No obstante, los resultados indican que el ciclo político no desapareció del todo.

Para reducir la cultura de la política como negocio, se propone reformar el artículo 41 de la Constitución por dos motivos básicos: uno es imponer un límite a los recursos destinados a gastos electorales y el otro es que el financiamiento de los partidos deje de ser exclusivamente público, con la intención de crear incentivos que obliguen a los políticos a trabajar por el bienestar de la sociedad. Otra propuesta es la posibilidad de que los políticos se reelijan como premio por un buen desempeño en el gobierno.

El análisis se hizo con variables reales porque es necesario conocer el nivel de bienestar social de las personas, ya que si los votantes ven una mejora en su economía doméstica reflejada en un mayor consumo de bienes y servicios con precios estables en el tiempo, favorecerán a los gobernantes en turno. Pero si sucede lo contrario, los castigarán no votando por ellos más adelante, además de que su mala gestión en la economía también repercutirá en los recursos públicos que reciban para las campañas electorales. Por esa razón, se busca anclar el monto de los recursos al nivel de crecimiento real de la economía, con el fin de que sea un incentivo para tener un buen desempeño por parte de los gobernantes.

En cuanto a la política monetaria se recomienda seguir manteniendo la autonomía del banco central así como su objetivo prioritario, que es preservar la estabilidad del poder adquisitivo de la moneda nacional e instrumentar las herramientas de política monetaria que crea convenientes para el logro de sus metas. Por último, respecto a la política cambiaria, se recomienda que en la Comisión de Cambios el voto de calidad sea transferido a los funcionarios del Banco de México, para evitar cualquier tentación de volver a manipular la paridad cambiaria. 
Algunos elementos que explican el menor impacto del ciclo político en la economía mexicana en fecha recientes son los siguientes: 1) una mayor apertura comercial, que puede limitar y generar filtraciones en una política fiscal y monetaria expansiva; 2) menor impacto de la política monetaria ante la autonomía alcanzada por el Banco de México, que le permite resistir las peticiones o, incluso, las presiones de los gobiernos en turno para instrumentar una política monetaria expansiva; 3) mayor transparencia y monitoreo de los recursos públicos, dada la alternancia en el poder, circunstancia que genera una mayor vigilancia y que limita a los políticos en el uso discrecional de dichos recursos; 4) la competencia política y la alternancia electoral en el nivel federal han causado que el ciclo político migre al ámbito estatal y municipal situación que se refleja en los recientes incrementos en el nivel de endeudamiento y gasto público de algunos estados y municipios, como lo ejemplifica el caso de Coahuila, y 5) finalmente, esta situación ha generado que el Congreso ponga límites al endeudamiento de los estados y que haya una mayor transparencia en la contratación de deuda, lo que podría dificultar su desvío con fines electorales, sin que esto anule la necesidad de etiquetar la mayoría de los recursos que provienen de la Federación para eliminar el uso discrecional de dichos recursos. En este contexto, queda claro que hay un amplio camino por recorrer para reducir los efectos negativos de tener un ciclo político en la economía mexicana.

\section{Anexo eStadístico}

Cuadro A.1. Prueba de estabilidad de Chow

\begin{tabular}{|c|l|c|c|c|}
\hline \multicolumn{1}{|c|}{$1981_{4}-1983_{1}$} \\
\hline \multirow{2}{*}{$P I B$} & Prueba $F$ & 18.85757 & Probabilidad & 0.000000 \\
\cline { 2 - 5 } & Tasa de verosimilitud & 65.36084 & Probabilidad & 0.000000 \\
\hline \multirow{2}{*}{$G P$} & Prueba $F$ & 5.909468 & Probabilidad & 0.000169 \\
\cline { 2 - 5 } & Tasa de verosimilitud & 22.96992 & Probabilidad & 0.000128 \\
\hline \multirow{2}{*}{$B M$} & Prueba $F$ & 28.11043 & Probabilidad & 0.000000 \\
\cline { 2 - 5 } & Tasa de verosimilitud & 90.78298 & Probabilidad & 0.000000 \\
\hline \multirow{2}{*}{$I N F$} & Prueba $F$ & 71.44056 & Probabilidad & 0.000000 \\
\cline { 2 - 5 } & Tasa de verosimilitud & 178.6999 & Probabilidad & 0.000000 \\
\hline
\end{tabular}

Fuente: Elaboración propia con el programa Eviews 5. 
222 ECONOMÍA: TEORÍA Y PRÁCTICA • Nueva Época, número 40, enero-junio 2014

Cuadro A.2. Pruebas de diagnóstico de mínimos cuadrados ordinarios, 1960-1983

\begin{tabular}{|l|c|}
\hline \multicolumn{1}{|c|}{ Prueba } & Probabilidad \\
\hline Jarque-Bera & 1.039534 \\
\hline Probabilidad (JB) & 0.594659 \\
\hline Durbin-Watson & $1.995>1.932<(4-1.995)$ \\
\hline LM (3) & 0.119068 \\
\hline ARCH (3) & 0.574292 \\
\hline White (sin términos cruzados) & 0.992702 \\
\hline Ramsey RESET (3) & 0.247719 \\
\hline
\end{tabular}

Fuente: Elaboración propia con el programa Eviews 5.

Cuadro A.3. Pruebas de diagnóstico de mínimos cuadrados ordinarios, 1984-2011

\begin{tabular}{|l|c|}
\hline \multicolumn{1}{|c|}{ Prueba } & Probabilidad \\
\hline Jarque-Bera & 4.520310 \\
\hline Probabilidad (JB) & 0.104334 \\
\hline Durbin-Watson & $1.850<2.014<(4-1.850)$ \\
\hline LM (3) & 0.152981 \\
\hline ARCH (3) & 0.483459 \\
\hline White (sin términos cruzados) & 0.902770 \\
\hline Ramsey RESET (3) & 0.106849 \\
\hline
\end{tabular}

Fuente: Elaboración propia con el programa Eviews 5.

Cuadro A.4. Pruebas de diagnóstico de vectores autorregresivos, 1960-1983 (90 observaciones)

\begin{tabular}{|l|c|l|c|l|l|}
\hline \multicolumn{2}{|c|}{ Autocorrelación* } & \multicolumn{2}{c|}{ Normalidad conjunta } & \multicolumn{2}{c|}{ Heterocedasticidad** } \\
\hline Rezagos & 6 & Jarque-Bera & 13.174050 & $x^{2}$ & 510.316700 \\
\hline LM & 9.569889 & $\begin{array}{l}\text { Grados de } \\
\text { libertad }\end{array}$ & 8 & $\begin{array}{l}\text { Grados de } \\
\text { libertad }\end{array}$ & 540 \\
\hline Probabilidad & 0.888100 & Probabilidad & 0.106000 & Probabilidad & 0.815900 \\
\hline
\end{tabular}

* Probabilidad de $X^{2}$ con 16 grados de libertad.

** Tests: sin términos cruzados (sólo niveles y cuadrados).

Fuente: elaboración propia con el programa Eviews 5. 
Cuadro A.5. Pruebas de diagnóstico de vectores autorregresivos, 1984-2011 (112 observaciones)

\begin{tabular}{|l|c|l|c|c|c|}
\hline \multicolumn{2}{|c|}{ Autocorrelación * } & \multicolumn{2}{c|}{ Normalidad conjunta } & \multicolumn{2}{c|}{ Heterocedasticidad ** } \\
\hline Rezagos & 6 & Jarque-Bera & 9.525178 & $x^{2}$ & 581.915900 \\
\hline LM & 22.129590 & $\begin{array}{l}\text { Grados de } \\
\text { libertad }\end{array}$ & 8 & $\begin{array}{c}\text { Grados de } \\
\text { libertad }\end{array}$ & 530 \\
\hline Probabilidad & 0.139100 & Probabilidad & 0.299900 & Probabilidad & 0.058700 \\
\hline
\end{tabular}

* Probabilidad de $X^{2}$ con 16 grados de libertad.

** Tests: sin términos cruzados (sólo niveles y cuadrados).

Fuente: Elaboración propia con el programa Eviews 5.

\section{Cuadro A.6. Vectores autorregresivos, $1961_{3}-1983_{4}$ (continúa)}

Período (ajustado): $1961_{3}-1983_{4}$

Observaciones: 90 después de ajuste

Errores estándar en ( ). Prueba $t$ en [ ].

\begin{tabular}{|c|c|c|c|c|}
\hline & $P I B$ & $G P$ & $B M$ & INF \\
\hline \multirow[t]{3}{*}{$P I B(-1)$} & 0.85296 & -0.15192 & 0.273834 & -0.403475 \\
\hline & -0.11374 & -0.39853 & -0.21981 & -0.17405 \\
\hline & [ 7.49891] & {$[-0.38120]$} & [ 1.24580$]$ & {$[-2.31815]$} \\
\hline \multirow[t]{3}{*}{$P I B(-2)$} & -0.081113 & -0.172323 & -0.221789 & 0.128229 \\
\hline & -0.1422 & -0.49824 & -0.2748 & -0.2176 \\
\hline & {$[-0.57040]$} & {$[-0.34586]$} & [- 0.80708] & [ 0.58929$]$ \\
\hline \multirow[t]{3}{*}{$P I B(-3)$} & 0.128425 & 0.667329 & 0.310145 & -0.285712 \\
\hline & -0.13972 & -0.48954 & -0.27 & -0.2138 \\
\hline & [ 0.91916$]$ & [ 1.36318$]$ & [ 1.14868] & {$[-1.33637]$} \\
\hline \multirow[t]{3}{*}{$P I B(-4)$} & -0.533617 & 0.682845 & -0.416192 & 0.272727 \\
\hline & -0.15329 & -0.5371 & -0.29623 & -0.23457 \\
\hline & [- 3.48102] & [ 1.27137$]$ & [- 1.40495$]$ & [ 1.16268$]$ \\
\hline \multirow[t]{3}{*}{$P I B(-5)$} & 0.329533 & -1.712498 & -0.155196 & 0.608546 \\
\hline & -0.17777 & -0.62284 & -0.34352 & -0.27201 \\
\hline & [ 1.85376$]$ & {$[-2.74951]$} & [- 0.45178$]$ & [ 2.23718$]$ \\
\hline \multirow[t]{3}{*}{$P I B(-6)$} & - 0.038789 & 0.197136 & 0.158757 & -0.146421 \\
\hline & -0.15221 & -0.53331 & -0.29414 & -0.23291 \\
\hline & {$[-0.25484]$} & [ 0.36965$]$ & [ 0.53973] & {$[-0.62865]$} \\
\hline
\end{tabular}

\begin{tabular}{|c|c|c|c|c|}
\hline & $P I B$ & $G P$ & $B M$ & INF \\
\hline \multirow[t]{3}{*}{$G P(-1)$} & -0.002856 & 0.988038 & 0.00503 & -0.009768 \\
\hline & -0.01201 & -0.04207 & -0.0232 & -0.01837 \\
\hline & {$[-0.23785]$} & [ 23.4876] & {$[0.21678]$} & {$[-0.53169]$} \\
\hline \multirow[t]{3}{*}{$G P(-2)$} & 0.004738 & 0.002004 & -0.004162 & 0.010382 \\
\hline & -0.01361 & -0.04767 & -0.02629 & -0.02082 \\
\hline & [ 0.34822$]$ & [ 0.04203$]$ & {$[-0.15830]$} & [ 0.49863$]$ \\
\hline \multirow[t]{3}{*}{$G P(-3)$} & -0.002194 & 0.011611 & 0.006895 & -0.004049 \\
\hline & -0.01164 & -0.04079 & -0.0225 & -0.01781 \\
\hline & {$[-0.18848]$} & [ 0.28465$]$ & {$[0.30646]$} & {$[-0.22726]$} \\
\hline \multirow[t]{3}{*}{$G P(-4)$} & 0.006783 & -0.198761 & 0.000496 & 0.014279 \\
\hline & -0.01338 & -0.04687 & -0.02585 & -0.02047 \\
\hline & [ 0.50704$]$ & {$[-4.24078]$} & {$[0.01919]$} & [ 0.69759$]$ \\
\hline \multirow[t]{3}{*}{$G P(-5)$} & -0.002845 & 0.188767 & -0.004915 & -0.020109 \\
\hline & -0.01368 & -0.04793 & -0.02643 & -0.02093 \\
\hline & {$[-0.20800]$} & [ 3.93874$]$ & {$[-0.18596]$} & {$[-0.96074]$} \\
\hline \multirow[t]{3}{*}{$G P(-6)$} & -0.00447 & -0.021841 & -0.021107 & 0.007425 \\
\hline & -0.00993 & -0.03481 & -0.0192 & -0.0152 \\
\hline & {$[-0.44995]$} & {$[-0.62749]$} & {$[-1.09945]$} & [ 0.48844$]$ \\
\hline
\end{tabular}


224 ECONOMÍA: TEORÍA Y PRÁCTICA • Nueva Época, número 40, enero-junio 2014

Cuadro A.6. Vectores autorregresivos, $1961_{3}-1983_{4}$ (continúa)

\begin{tabular}{|c|c|c|c|c|c|c|c|c|c|}
\hline \multirow{3}{*}{$B M(-1)$} & $P I B$ & $G P$ & $B M$ & $I N F$ & \multirow[b]{2}{*}{$I N F(-5)$} & $P I B$ & $G P$ & $B M$ & $I N F$ \\
\hline & -0.006029 & -0.420079 & 0.684682 & 0.331534 & & -0.033495 & -1.047386 & -0.410683 & 0.764692 \\
\hline & -0.05793 & -0.20298 & -0.11195 & -0.08865 & & -0.15212 & -0.533 & -0.29397 & -0.23278 \\
\hline \multirow{4}{*}{$B M(-2)$} & {$[-0.10408]$} & {$[-2.06959]$} & [ 6.11591] & [ 3.73994] & & {$[-0.22018]$} & {$[-1.96508]$} & {$[-1.39701]$} & [ 3.28507] \\
\hline & 0.023222 & 0.068724 & -0.009367 & 0.012354 & $\operatorname{INF}(-6)$ & 0.065873 & 0.177327 & 0.30928 & -0.34911 \\
\hline & -0.07749 & -0.27149 & -0.14974 & -0.11857 & & -0.10254 & -0.35927 & -0.19816 & -0.15691 \\
\hline & [ 0.29969$]$ & {$[0.25313]$} & {$[-0.06255]$} & {$[0.10419]$} & & [ 0.64241$]$ & [ 0.49357$]$ & [ 1.56080$]$ & {$[-2.22495]$} \\
\hline \multirow[t]{3}{*}{$B M(-3)$} & -0.044252 & 0.009419 & -0.026892 & 0.066481 & c & 0.012404 & 0.061266 & 0.052289 & -0.035593 \\
\hline & -0.06891 & -0.24146 & -0.13317 & -0.10545 & & -0.00842 & -0.02949 & -0.01626 & -0.01288 \\
\hline & {$[-0.64214]$} & {$[0.03901]$} & {$[-0.20193]$} & {$[0.63044]$} & & [ 1.47398] & [ 2.07784] & [ 3.21529] & {$[-2.76405]$} \\
\hline \multirow[t]{3}{*}{$B M(-4)$} & 0.085732 & 0.376021 & -0.327281 & 0.142901 & $E L E C$ & -0.004811 & -0.010499 & -0.015479 & 0.016902 \\
\hline & -0.07235 & -0.2535 & -0.13982 & -0.11071 & & -0.00535 & -0.01875 & -0.01034 & -0.00819 \\
\hline & [ 1.18492$]$ & [ 1.48330$]$ & {$[-2.34075]$} & [ 1.29073$]$ & & {$[-0.89897]$} & {$[-0.55990]$} & {$[-1.49675]$} & [ 2.06391] \\
\hline \multirow[t]{3}{*}{$B M(-5)$} & 0.046527 & -0.078651 & 0.532377 & -0.506905 & $D 64$ & 0.040156 & 0.315876 & -0.000397 & 0.000318 \\
\hline & -0.07537 & -0.26408 & -0.14565 & -0.11533 & & -0.01547 & -0.0542 & -0.0299 & -0.02367 \\
\hline & [ 0.61731$]$ & {$[-0.29784]$} & [ 3.65519] & {$[-4.39523]$} & & [ 2.59565$]$ & [ 5.82751] & {$[-0.01328]$} & [ 0.01342$]$ \\
\hline \multirow[t]{3}{*}{$B M(-6)$} & 0.025464 & -0.182615 & -0.280741 & 0.214811 & $D 65$ & -0.03497 & 0.89427 & -0.078871 & 0.001049 \\
\hline & -0.07384 & -0.25872 & $\mid-0.1427$ & -0.11299 & & -0.01612 & -0.05646 & -0.03114 & -0.02466 \\
\hline & [ 0.34484$]$ & {$[-0.70584]$} & [- 1.96742] & [ 1.90113$]$ & & [- 2.16993] & [ 15.8378] & [- 2.53256] & [ 0.04255$]$ \\
\hline \multirow[t]{3}{*}{$I N F(-1)$} & -0.194926 & -0.326686 & -0.645779 & 1.757448 & $D 66$ & -0.022516 & -0.93497 & 0.004463 & -0.020063 \\
\hline & -0.08338 & -0.29212 & -0.16112 & -0.12758 & & -0.02076 & -0.07275 & -0.04012 & -0.03177 \\
\hline & {$[-2.33792]$} & {$[-1.11831]$} & {$[-4.00806]$} & [ 13.7752] & & {$[-1.08443]$} & {$[-12.8522]$} & [ 0.11123] & {$[-0.63149]$} \\
\hline \multirow[t]{3}{*}{ INF(- 2) } & 0.225754 & -0.283626 & 0.658669 & -0.717794 & $D 80$ & -0.016458 & 0.272078 & -0.011886 & 0.01869 \\
\hline & -0.13987 & -0.49008 & -0.2703 & -0.21403 & & -0.01517 & -0.05314 & -0.02931 & -0.02321 \\
\hline & [ 1.61398$]$ & {$[-0.57874]$} & [ 2.43680] & {$[-3.35364]$} & & [- 1.08509] & [ 5.11995] & {$[-0.40552]$} & [ 0.80533] \\
\hline \multirow[t]{3}{*}{$I N F(-3)$} & -0.14217 & 0.192624 & -0.294144 & 0.306847 & $D 82$ & -0.048515 & 0.520436 & 0.194363 & 0.01171 \\
\hline & -0.12634 & -0.44264 & -0.24414 & -0.19332 & & -0.01744 & -0.0611 & -0.0337 & -0.02668 \\
\hline & {$[-1.12534]$} & [ 0.43517] & {$[-1.20483]$} & [ 1.58727$]$ & & [- 2.78208] & [ 8.51793] & [ 5.76766] & [ 0.43885] \\
\hline \multirow[t]{3}{*}{$\operatorname{INF}(-4)$} & 0.104583 & 1.185786 & 0.325035 & -0.738003 & & & & & \\
\hline & -0.14383 & -0.50394 & -0.27794 & -0.22009 & & & & & \\
\hline & [ 0.72713] & [ 2.35304] & [ 1.16943$]$ & {$[-3.35324]$} & & & & & \\
\hline
\end{tabular}


Cuadro A.6. Vectores autorregresivos, $1961_{3}-1983_{4}$ (concluye)

\begin{tabular}{|c|c|c|c|c|c|c|c|c|c|}
\hline & $P I B$ & GP & $B M$ & INF & & $P I B$ & GP & $B M$ & INF \\
\hline$R^{2}$ & 0.883423 & 0.976111 & 0.949136 & 0.994416 & $\begin{array}{l}\text { Significación } \\
\text { de la } \\
\text { variable } \\
\text { dependiente }\end{array}$ & 0.059678 & 0.161811 & 0.062156 & 0.168611 \\
\hline$R^{2}$ ajustada & 0.824146 & 0.963964 & 0.923273 & 0.991577 & $\begin{array}{l}\text { Desviación } \\
\text { estándar de } \\
\text { la variable } \\
\text { dependiente }\end{array}$ & 0.034082 & 0.263792 & 0.099710 & 0.238288 \\
\hline $\begin{array}{l}\text { Suma de } \\
\text { cuadrados de } \\
\text { los residuos }\end{array}$ & 0.012052 & 0.147950 & 0.045006 & 0.028219 & \multicolumn{4}{|c|}{$\begin{array}{l}\text { Determinante de la covarianza residual } \\
\text { (grados de libertad ajustados) }\end{array}$} & 0.000000 \\
\hline $\begin{array}{l}\text { Ecuación } \\
\text { de error } \\
\text { estándar }\end{array}$ & 0.014292 & 0.050076 & 0.027619 & 0.021870 & \multicolumn{4}{|c|}{ Determinante de la covarianza residual } & 0.000000 \\
\hline Prueba $F$ & 14.903420 & 80.358180 & 36.698630 & 350.226100 & \multicolumn{4}{|c|}{ Función de verosimil } & 917.869600 \\
\hline $\begin{array}{l}\text { Función de } \\
\text { verosimilitud }\end{array}$ & 273.620800 & 160.776700 & 214.329700 & 235.335700 & \multicolumn{4}{|c|}{$\begin{array}{l}\text { Criterio de información } \\
\text { de Akaike }\end{array}$} & -17.641550 \\
\hline $\begin{array}{l}\text { Criterio de } \\
\text { información } \\
\text { de Akaike }\end{array}$ & -5.391574 & -2.883928 & -4.073993 & -4.540792 & \multicolumn{4}{|c|}{ Criterio de Schwarz } & -14.197360 \\
\hline $\begin{array}{l}\text { Criterio } \\
\text { de Schwarz }\end{array}$ & -4.530528 & -2.022882 & -3.212947 & -3.679747 & & & & & \\
\hline
\end{tabular}

Fuente: Elaboración propia con el programa Eviews 5.

\section{Cuadro A.7. Vectores autorregresivos, $1984_{1}-2011_{4}$} (continúa)

Periodo: $1984_{1}-2011_{4}$

Observaciones: 112

Errores estándar en ( ). Prueba $t$ en [ ].

\begin{tabular}{|c|c|c|c|c|c|c|c|c|c|}
\hline \multirow[b]{2}{*}{$P I B(-1)$} & $P I B$ & $G P$ & $B M$ & INF & \multirow[b]{2}{*}{ PIB(- 4) } & $P I B$ & $G P$ & $B M$ & INF \\
\hline & 0.897223 & 0.120746 & 0.306692 & 0.348309 & & -0.168068 & 0.061145 & 0.237172 & 0.389877 \\
\hline & -0.10844 & -0.24537 & -0.28832 & -0.25663 & & -0.137 & -0.30999 & -0.36425 & -0.32421 \\
\hline & [ 8.27388] & [ 0.49209] & [ 1.06371$]$ & [ 1.35723] & & {$[-1.22680]$} & [ 0.19725$]$ & [ 0.65112$]$ & [ 1.20253] \\
\hline \multirow[t]{3}{*}{$P I B(-2)$} & -0.027891 & -0.138669 & 0.184782 & -0.108065 & $P I B(-5)$ & 0.292737 & -0.153345 & 0.134287 & -0.34066 \\
\hline & -0.14769 & -0.33418 & -0.39268 & -0.34951 & & -0.13284 & -0.30058 & -0.3532 & -0.31437 \\
\hline & {$[-0.18885]$} & [- 0.41495] & [ 0.47057] & {$[-0.30919]$} & & [ 2.20368] & {$[-0.51016]$} & {$[0.38020]$} & {$[-1.08361]$} \\
\hline \multirow[t]{3}{*}{$P I B(-3)$} & -0.248587 & -0.137088 & -0.572143 & -0.263654 & $P I B(-6)$ & -0.14492 & -0.00512 & 0.007624 & 0.255165 \\
\hline & -0.14384 & -0.32548 & -0.38246 & -0.34042 & & -0.10164 & -0.22998 & -0.27023 & -0.24053 \\
\hline & {$[-1.72817]$} & {$[-0.42118]$} & [- 1.49597] & {$[-0.77450]$} & & {$[-1.42587]$} & {$[-0.02226]$} & {$[0.02821]$} & [ 1.06084$]$ \\
\hline
\end{tabular}


226 ECONOMÍA: TEORÍA Y PRÁCTICA • Nueva Época, número 40, enero-junio 2014

Cuadro A.7. Vectores autorregresivos, $1984_{1}-2011_{4}$ (continúa)

\begin{tabular}{|c|c|c|c|c|c|c|c|c|c|}
\hline & $P I B$ & $G P$ & $B M$ & $I N F$ & & PIB & $G P$ & $B M$ & $I N F$ \\
\hline \multirow[t]{3}{*}{$G P(-1)$} & -0.021311 & 0.760095 & -0.149948 & 0.199059 & $B M(-4)$ & 0.030576 & 0.183476 & -0.489133 & 0.071395 \\
\hline & -0.03026 & -0.06848 & -0.08047 & -0.07162 & & -0.04481 & -0.1014 & -0.11915 & -0.10605 \\
\hline & [- 0.70414] & [ 11.0994] & {$[-1.86344]$} & [ 2.77924$]$ & & [ 0.68231$]$ & [ 1.80946$]$ & {$[-4.10526]$} & [ 0.67321$]$ \\
\hline \multirow[t]{3}{*}{$G P(-2)$} & -0.012369 & 0.053314 & 0.022184 & -0.012742 & $B M(-5)$ & 0.010756 & -0.18751 & -0.054106 & 0.019428 \\
\hline & -0.03891 & -0.08805 & -0.10347 & -0.09209 & & -0.04327 & -0.0979 & -0.11504 & -0.10239 \\
\hline & [- 0.31784$]$ & [ 0.60548$]$ & {$[0.21441]$} & {$[-0.13836]$} & & {$[0.24860]$} & [- 1.91530] & {$[-0.47033]$} & [ 0.18973] \\
\hline \multirow[t]{3}{*}{$G P(-3)$} & 0.00519 & 0.016653 & 0.062061 & -0.052418 & $B M(-6)$ & -0.03528 & 0.02199 & 0.198832 & -0.058343 \\
\hline & -0.03786 & -0.08568 & -0.10067 & -0.08961 & & -0.03807 & -0.08614 & - 0.10122 & -0.09009 \\
\hline & [ 0.13707$]$ & [ 0.19437$]$ & [ 0.61646$]$ & {$[-0.58497]$} & & {$[-0.92673]$} & [ 0.25529] & [ 1 1.96438] & {$[-0.64758]$} \\
\hline \multirow{3}{*}{$G P(-4)$} & 0.019316 & -0.304315 & 0.186411 & -0.062681 & $I N F(-1)$ & 0.068791 & -0.051472 & -0.104924 & 1.789224 \\
\hline & -0.04309 & -0.09751 & -0.11458 & -0.10198 & & -0.05215 & -0.11801 & -0.13867 & -0.12343 \\
\hline & [ 0.44823] & [- 3.12086] & [ 1.62693$]$ & {$[-0.61461]$} & & [ 1.31900$]$ & [- 0.43616] & {$[-0.75666]$} & [ 14.4963] \\
\hline \multirow[t]{3}{*}{$G P(-5)$} & -0.008983 & 0.232617 & -0.082704 & 0.123672 & $I N F(-2)$ & -0.206294 & -0.133024 & 0.004204 & -0.876634 \\
\hline & -0.04329 & -0.09796 & -0.11511 & -0.10246 & & -0.0945 & -0.21383 & -0.25126 & -0.22364 \\
\hline & {$[-0.20749]$} & [ 2.37449] & {$[-0.71846]$} & [ 1.20702$]$ & & {$[-2.18297]$} & {$[-0.62210]$} & [ 0.01673] & [- 3.91976] \\
\hline \multirow[t]{3}{*}{$G P(-6)$} & -0.028449 & 0.003772 & -0.154531 & 0.069888 & INF(- 3) & 0.213098 & 0.661033 & -0.173547 & 0.240398 \\
\hline & -0.03069 & -0.06945 & -0.0816 & -0.07263 & & -0.10073 & -0.22793 & -0.26783 & -0.23839 \\
\hline & [- 0.92695] & [ 0.05432] & {$[-1.89373]$} & [ 0.96222$]$ & & [ 2.11550$]$ & [ 2.90017] & {$[-0.64798]$} & [ 1.00843] \\
\hline \multirow[t]{3}{*}{$B M(-1)$} & 0.096104 & 0.078261 & 0.408665 & 0.067691 & $I N F(-4)$ & -0.054202 & -0.863437 & 0.643858 & -0.696584 \\
\hline & -0.04311 & -0.09754 & -0.11461 & -0.10201 & & -0.10353 & -0.23426 & - 0.27527 & -0.24501 \\
\hline & [ 2.22945] & [ 0.80235$]$ & [ 3.56563] & [ 0.66354$]$ & & {$[-0.52354]$} & {$[-3.68581]$} & [ 2.33904$]$ & [- 2.84309] \\
\hline \multirow[t]{3}{*}{$B M(-2)$} & -0.133071 & -0.108724 & 0.274574 & 0.032036 & INF(- 5) & -0.039393 & 0.261335 & -0.535893 & 0.790762 \\
\hline & -0.04858 & -0.10991 & -0.12915 & -0.11496 & & -0.09005 & -0.20375 & -0.23942 & -0.2131 \\
\hline & [- 2.73948] & [- 0.98918] & [ 2.12597$]$ & [ 0.27868$]$ & & {$[-0.43747]$} & [ 1.28260$]$ & {$[-2.23830]$} & [ 3.71070$]$ \\
\hline \multirow[t]{3}{*}{$B M(-3)$} & 0.00455 & -0.033389 & 0.102341 & -0.084967 & INF(- 6) & 0.01181 & 0.1031 & 0.061966 & -0.233178 \\
\hline & -0.04537 & -0.10266 & -0.12063 & -0.10737 & & -0.04542 & -0.10277 & -0.12076 & -0.10748 \\
\hline & [ 0.10028$]$ & [- 0.32524] & [ 0.84838] & [- 0.79134] & & {$[0.26003]$} & [ 1.00323] & {$[0.51315]$} & [- 2.16944] \\
\hline
\end{tabular}


Cuadro A.7. Vectores autorregresivos, 1984,-2011 (concluye)

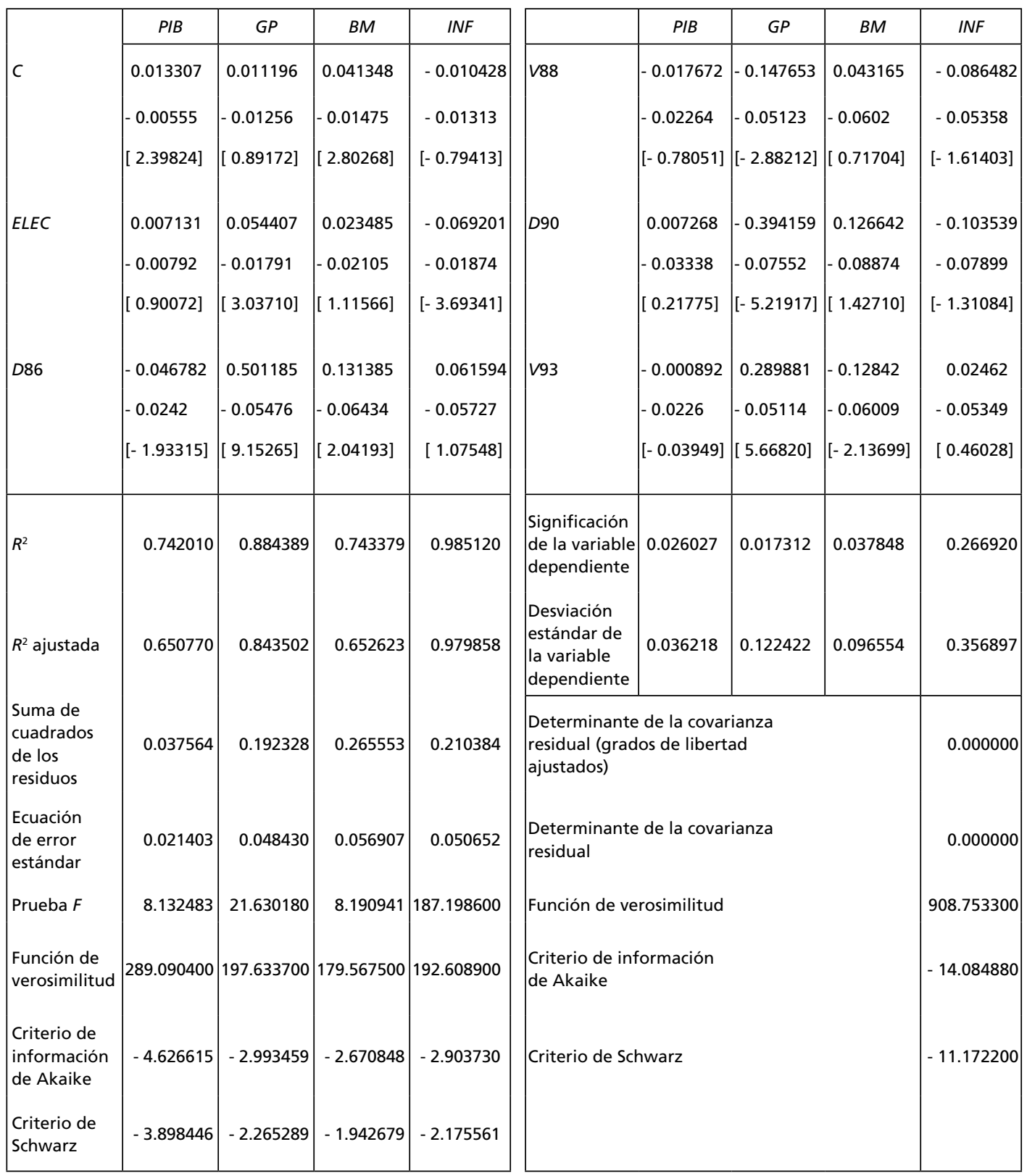

Fuente: Elaboración propia con el programa Eviews 5. 
228 ECONOMÍA: TEORÍA Y PRÁCTICA • Nueva Época, número 40, enero-junio 2014

\section{Cuadro A.8. Series históricas}

(cifras en millones de pesos a precios de 2003)

(continúa)

\begin{tabular}{|c|c|c|c|c|c|c|c|c|c|c|}
\hline \multirow{2}{*}{\multicolumn{2}{|c|}{ Temporalidad }} & \multicolumn{2}{|c|}{$\mathrm{PIB}^{*}$} & \multicolumn{2}{|c|}{ Base monetaria** } & \multirow{2}{*}{\multicolumn{2}{|c|}{$\begin{array}{c}\text { Egresos del } \\
\text { sector público } \\
\text { presupuestario*** } \\
\text { Flujos acumulados }\end{array}$}} & \multicolumn{2}{|c|}{$\operatorname{INPC} * * * *$} & \multirow{3}{*}{ 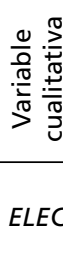 } \\
\hline & & \multicolumn{2}{|c|}{ Precios de mercado } & \multicolumn{2}{|c|}{$\begin{array}{l}\text { Saldos acumulados al } \\
\text { cierre del trimestre }\end{array}$} & & & Base 200 & $3_{1}=100$ & \\
\hline Año & Trimestre & Absolutos & $\begin{array}{c}\text { Variación } \\
\text { anual } \\
(\%)\end{array}$ & Absolutos & $\begin{array}{c}\text { Variación } \\
\text { anual } \\
(\%)\end{array}$ & Absolutos & $\begin{array}{c}\text { Variación } \\
\text { anual } \\
(\%)\end{array}$ & Índice & $\begin{array}{c}\text { Variación } \\
\text { anual } \\
(\%)\end{array}$ & \\
\hline 1959 & 1 & 1131994.3 & & 43227.7 & & 16453.9 & & 0.017119 & & 0 \\
\hline 1959 & 2 & 1137500.4 & & 50339.8 & & 34988.8 & & 0.017084 & & 0 \\
\hline 1959 & 3 & 1091785.7 & & 51263.1 & & 56666.6 & & 0.016971 & & 0 \\
\hline 1959 & 4 & 1152858.5 & & 71520.9 & & 83245.7 & & 0.017058 & & 0 \\
\hline 1960 & 1 & 1223777.7 & 8.1 & 46796.7 & 8.3 & 23149.0 & 40.7 & 0.017309 & 1.1 & 0 \\
\hline 1960 & 2 & 1229730.2 & 8.1 & 52501.1 & 4.3 & 47492.0 & 35.7 & 0.017904 & 4.8 & 0 \\
\hline 1960 & 3 & 1180308.9 & 8.1 & 52551.7 & 2.5 & 75677.7 & 33.5 & 0.018077 & 6.5 & 0 \\
\hline 1960 & 4 & 1246333.6 & 8.1 & 74716.7 & 4.5 & 112632.6 & 35.3 & 0.017934 & 5.1 & 0 \\
\hline 1961 & 1 & 1276636.0 & 4.3 & 47671.4 & 1.9 & 22430.5 & -3.1 & 0.018040 & 4.2 & 0 \\
\hline 1961 & 2 & 1282845.6 & 4.3 & 55193.4 & 5.1 & 47396.4 & -0.2 & 0.018118 & 1.2 & 0 \\
\hline 1961 & 3 & 1231289.7 & 4.3 & 56364.5 & 7.3 & 77102.3 & 1.9 & 0.017919 & -0.9 & 0 \\
\hline 1961 & 4 & 1300166.2 & 4.3 & 79996.0 & 7.1 & 114120.1 & 1.3 & 0.017876 & -0.3 & 0 \\
\hline 1962 & 1 & 1333557.7 & 4.5 & 53628.4 & 12.5 & 22152.6 & -1.2 & 0.018087 & 0.3 & 0 \\
\hline 1962 & 2 & 1340044.2 & 4.5 & 61487.2 & 11.4 & 46268.5 & -2.4 & 0.018378 & 1.4 & 0 \\
\hline 1962 & 3 & 1286189.5 & 4.5 & 61463.0 & 9.0 & 73758.8 & -4.3 & 0.018548 & 3.5 & 0 \\
\hline 1962 & 4 & 1358137.0 & 4.5 & 86946.8 & 8.7 & 109088.6 & -4.4 & 0.018517 & 3.6 & 0 \\
\hline 1963 & 1 & 1434136.1 & 7.5 & 61245.2 & 14.2 & 21824.3 & -1.5 & 0.018450 & 2.0 & 0 \\
\hline 1963 & 2 & 1441111.8 & 7.5 & 71307.3 & 16.0 & 46162.0 & -0.2 & 0.018511 & 0.7 & 0 \\
\hline 1963 & 3 & 1383195.3 & 7.5 & 71965.5 & 17.1 & 74391.2 & 0.9 & 0.018481 & -0.4 & 0 \\
\hline 1963 & 4 & 1460569.2 & 7.5 & 102059.2 & 17.4 & 110202.2 & 1.0 & 0.018421 & -0.5 & 0 \\
\hline 1964 & 1 & 1592054.4 & 11.0 & 69678.3 & 13.8 & 29631.9 & 35.8 & 0.018944 & 2.7 & 0 \\
\hline 1964 & 2 & 1599798.2 & 11.0 & 80299.3 & 12.6 & 62116.3 & 34.6 & 0.019178 & 3.6 & 1 \\
\hline 1964 & 3 & 1535504.4 & 11.0 & 79830.8 & 10.9 & 98713.9 & 32.7 & 0.019416 & 5.1 & 1 \\
\hline 1964 & 4 & 1621398.2 & 11.0 & 112818.6 & 10.5 & 145788.4 & 32.3 & 0.019412 & 5.4 & 1 \\
\hline 1965 & 1 & 1689946.4 & 6.1 & 72135.5 & 3.5 & 64947.1 & 119.2 & 0.019547 & 3.2 & 0 \\
\hline 1965 & 2 & 1698166.4 & 6.1 & 83038.2 & 3.4 & 136408.7 & 119.6 & 0.019750 & 3.0 & 0 \\
\hline 1965 & 3 & 1629919.3 & 6.1 & 84383.7 & 5.7 & 220335.2 & 123.2 & 0.019672 & 1.3 & 0 \\
\hline 1965 & 4 & 1721094.5 & 6.1 & 119085.1 & 5.6 & 325702.8 & 123.4 & 0.019650 & 1.2 & 0 \\
\hline
\end{tabular}


Cuadro A.8. Series históricas

(cifras en millones de pesos a precios de 2003) (continúa)

\begin{tabular}{|c|c|c|c|c|c|c|c|c|c|c|}
\hline Año & Trimestre & Absolutos & \begin{tabular}{|c|} 
Variación \\
anual \\
$(\%)$
\end{tabular} & Absolutos & \begin{tabular}{|c|} 
Variación \\
anual \\
$(\%)$
\end{tabular} & Absolutos & \begin{tabular}{|c|} 
Variación \\
anual \\
$(\%)$
\end{tabular} & Índice & \begin{tabular}{|c|} 
Variación \\
anual \\
$(\%)$
\end{tabular} & ELEC \\
\hline 1966 & 1 & 1792967.9 & 6.1 & 79517.9 & 10.2 & 66833.1 & 2.9 & 0.019618 & 0.4 & 0 \\
\hline 1966 & 2 & 1801689.0 & 6.1 & 92323.3 & 11.2 & 141146.3 & 3.5 & 0.019713 & -0.2 & 0 \\
\hline 1966 & 3 & 1729281.4 & 6.1 & 92044.8 & 9.1 & 223942.2 & 1.6 & 0.019990 & 1.6 & 0 \\
\hline 1966 & 4 & 1826014.8 & 6.1 & 129245.9 & 8.5 & 328582.9 & 0.9 & 0.020117 & 2.4 & 0 \\
\hline 1967 & 1 & 1897944.9 & 5.9 & 82931.4 & 4.3 & 77383.7 & 15.8 & 0.020378 & 3.9 & 0 \\
\hline 1967 & 2 & 1907176.5 & 5.9 & 96915.6 & 5.0 & 164635.2 & 16.6 & 0.020327 & 3.1 & 0 \\
\hline 1967 & 3 & 1830529.5 & 5.9 & 97383.8 & 5.8 & 263483.8 & 17.7 & 0.020435 & 2.2 & 0 \\
\hline 1967 & 4 & 1932926.6 & 5.9 & 136989.5 & 6.0 & 387568.1 & 18.0 & 0.020513 & 2.0 & 0 \\
\hline 1968 & 1 & 2076793.5 & 9.4 & 92671.4 & 11.7 & 80265.3 & 3.7 & 0.020610 & 1.1 & 0 \\
\hline 1968 & 2 & 2086895.1 & 9.4 & 106560.2 & 10.0 & 167758.5 & 1.9 & 0.020927 & 3.0 & 0 \\
\hline 1968 & 3 & 2003025.4 & 9.4 & 107628.6 & 10.5 & 270186.8 & 2.5 & 0.020905 & 2.3 & 0 \\
\hline 1968 & 4 & 2115071.6 & 9.4 & 151448.5 & 10.6 & 398448.2 & 2.8 & 0.020931 & 2.0 & 0 \\
\hline 1969 & 1 & 2147791.2 & 3.4 & 100595.8 & 8.6 & 92677.3 & 15.5 & 0.020975 & 1.8 & 0 \\
\hline 1969 & 2 & 2158238.1 & 3.4 & 116610.6 & 9.4 & 195549.8 & 16.6 & 0.021096 & 0.8 & 0 \\
\hline 1969 & 3 & 2071501.3 & 3.4 & 116856.6 & 8.6 & 311482.2 & 15.3 & 0.021308 & 1.9 & 0 \\
\hline 1969 & 4 & 2187377.9 & 3.4 & 161526.4 & 6.7 & 450985.3 & 13.2 & 0.021730 & 3.8 & 0 \\
\hline 1970 & 1 & 2287450.9 & 6.5 & 106241.1 & 5.6 & 98434.4 & 6.2 & 0.022025 & 5.0 & 0 \\
\hline 1970 & 2 & 2298577.2 & 6.5 & 123127.8 & 5.6 & 207511.3 & 6.1 & 0.022172 & 5.1 & 1 \\
\hline 1970 & 3 & 2206200.3 & 6.5 & 122812.4 & 5.1 & 329387.1 & 5.7 & 0.022473 & 5.5 & 1 \\
\hline 1970 & 4 & 2329611.8 & 6.5 & 171389.6 & 6.1 & 481565.1 & 6.8 & 0.022697 & 4.4 & 1 \\
\hline 1971 & 1 & 2373515.6 & 3.8 & 109128.9 & 2.7 & 103869.4 & 5.5 & 0.023184 & 5.3 & 0 \\
\hline 1971 & 2 & 2385060.4 & 3.8 & 125737.9 & 2.1 & 217816.6 & 5.0 & 0.023462 & 5.8 & 0 \\
\hline 1971 & 3 & 2289207.9 & 3.8 & 125739.9 & 2.4 & 346919.6 & 5.3 & 0.023700 & 5.5 & 0 \\
\hline 1971 & 4 & 2417262.7 & 3.8 & 176074.8 & 2.7 & 507731.0 & 5.4 & 0.023910 & 5.3 & 0 \\
\hline 1972 & 1 & 2568827.6 & 8.2 & 126862.5 & 16.3 & 121968.5 & 17.4 & 0.024199 & 4.4 & 0 \\
\hline 1972 & 2 & 2581322.4 & 8.2 & 145774.6 & 15.9 & 255052.8 & 17.1 & 0.024558 & 4.7 & 0 \\
\hline 1972 & 3 & 2477582.4 & 8.2 & 145173.9 & 15.5 & 404143.8 & 16.5 & 0.024936 & 5.2 & 0 \\
\hline 1972 & 4 & 2616174.6 & 8.2 & 202210.1 & 14.8 & 589977.8 & 16.2 & 0.025221 & 5.5 & 0 \\
\hline 1973 & 1 & 2770766.2 & 7.9 & 147389.2 & 16.2 & 156205.0 & 28.1 & 0.025918 & 7.1 & 0 \\
\hline 1973 & 2 & 2784243.3 & 7.9 & 166317.2 & 14.1 & 320386.0 & 25.6 & 0.026816 & 9.2 & 0 \\
\hline 1973 & 3 & 2672348.1 & 7.9 & 159525.4 & 9.9 & 488933.2 & 21.0 & 0.028271 & 13.4 & 0 \\
\hline 1973 & 4 & 2821835.2 & 7.9 & 212657.3 & 5.2 & 683517.3 & 15.9 & 0.029860 & 18.4 & 0 \\
\hline 1974 & 1 & 2930828.6 & 5.8 & 143471.6 & -2.7 & 169222.4 & 8.3 & 0.032411 & 25.1 & 0 \\
\hline 1974 & 2 & 2945084.2 & 5.8 & 161559.0 & -2.9 & 346940.6 & 8.3 & 0.033548 & 25.1 & 0 \\
\hline 1974 & 3 & 2826725.0 & 5.8 & 157841.2 & -1.1 & 539371.6 & 10.3 & 0.034718 & 22.8 & 0 \\
\hline 1974 & 4 & 2984847.7 & 5.8 & 211452.0 & -0.6 & 756358.1 & 10.7 & 0.036557 & 22.4 & 0 \\
\hline 1975 & 1 & 3099189.6 & 5.7 & 149320.6 & 4.1 & 210430.9 & 24.4 & 0.037771 & 16.5 & 0 \\
\hline 1975 & 2 & 3114264.1 & 5.7 & 169230.6 & 4.7 & 433811.1 & 25.0 & 0.038882 & 15.9 & 0 \\
\hline 1975 & 3 & 2989105.8 & 5.7 & 165687.2 & 5.0 & 676144.7 & 25.4 & 0.040136 & 15.6 & 0 \\
\hline 1975 & 4 & 3156311.9 & 5.7 & 229037.1 & 8.3 & 978413.2 & 29.4 & 0.040954 & 12.0 & 0 \\
\hline
\end{tabular}


230 ECONOMÍA: TEORÍA Y PRÁCTICA • Nueva Época, número 40, enero-junio 2014

\section{Cuadro A.8. Series históricas}

(cifras en millones de pesos a precios de 2003)

(continúa)

\begin{tabular}{|c|c|c|c|c|c|c|c|c|c|c|}
\hline Año & Trimestre & Absolutos & \begin{tabular}{|c|} 
Variación \\
anual \\
$(\%)$
\end{tabular} & Absolutos & $\begin{array}{c}\text { Variación } \\
\text { anual } \\
(\%)\end{array}$ & Absolutos & \begin{tabular}{|c|} 
Variación \\
anual \\
$(\%)$
\end{tabular} & Índice & $\begin{array}{c}\text { Variación } \\
\text { anual } \\
(\%)\end{array}$ & ELEC \\
\hline 1976 & 1 & 3236094.5 & 4.4 & 172697.4 & 15.7 & 241462.5 & 14.7 & 0.042734 & 13.1 & 0 \\
\hline 1976 & 2 & 3251835.0 & 4.4 & 196391.5 & 16.0 & 499479.9 & 15.1 & 0.043841 & 12.8 & 1 \\
\hline 1976 & 3 & 3121147.9 & 4.4 & 192587.4 & 16.2 & 778992.2 & 15.2 & 0.045226 & 12.7 & 1 \\
\hline 1976 & 4 & 3295740.2 & 4.4 & 241040.5 & 5.2 & 1021917.3 & 4.4 & 0.050904 & 24.3 & 1 \\
\hline 1977 & 1 & 3345818.8 & 3.4 & 168926.2 & -2.2 & 262107.6 & 8.6 & 0.055290 & 29.4 & 0 \\
\hline 1977 & 2 & 3362093.0 & 3.4 & 188900.4 & -3.8 & 532495.5 & 6.6 & 0.057755 & 31.7 & 0 \\
\hline 1977 & 3 & 3226974.8 & 3.4 & 183000.2 & -5.0 & 821680.3 & 5.5 & 0.060218 & 33.1 & 0 \\
\hline 1977 & 4 & 3407486.9 & 3.4 & 248470.1 & 3.1 & 1168161.5 & 14.3 & 0.062543 & 22.9 & 0 \\
\hline 1978 & 1 & 3645501.9 & 9.0 & 189022.8 & 11.9 & 283564.9 & 8.2 & 0.065601 & 18.6 & 0 \\
\hline 1978 & 2 & 3663233.8 & 9.0 & 213254.7 & 12.9 & 581792.9 & 9.3 & 0.067853 & 17.5 & 0 \\
\hline 1978 & 3 & 3516013.1 & 9.0 & 207106.6 & 13.2 & 899725.5 & 9.5 & 0.070592 & 17.2 & 0 \\
\hline 1978 & 4 & 3712693.5 & 9.0 & 282611.0 & 13.7 & 1285941.8 & 10.1 & 0.072927 & 16.6 & 0 \\
\hline 1979 & 1 & 3999048.9 & 9.7 & 213769.4 & 13.1 & 325182.9 & 14.7 & 0.077280 & 17.8 & 0 \\
\hline 1979 & 2 & 4018500.4 & 9.7 & 240904.1 & 13.0 & 666358.3 & 14.5 & 0.080032 & 17.9 & 0 \\
\hline 1979 & 3 & 3857002.0 & 9.7 & 234487.9 & 13.2 & 1032295.7 & 14.7 & 0.083117 & 17.7 & 0 \\
\hline 1979 & 4 & 4072756.8 & 9.7 & 316006.6 & 11.8 & 1457403.5 & 13.3 & 0.086929 & 19.2 & 0 \\
\hline 1980 & 1 & 4306865.8 & 7.7 & 232199.5 & 8.6 & 449464.4 & 38.2 & 0.094703 & 22.5 & 0 \\
\hline 1980 & 2 & 4294588.6 & 6.9 & 256244.8 & 6.4 & 902061.4 & 35.4 & 0.100139 & 25.1 & 0 \\
\hline 1980 & 3 & 4247688.7 & 10.1 & 243138.0 & 3.7 & 1362214.2 & 32.0 & 0.106688 & 28.4 & 0 \\
\hline 1980 & 4 & 4570620.0 & 12.2 & 326163.1 & 3.2 & 1914423.7 & 31.4 & 0.112091 & 28.9 & 0 \\
\hline 1981 & 1 & 4662048.7 & 8.2 & 240967.1 & 3.8 & 452163.5 & 0.6 & 0.121178 & 28.0 & 0 \\
\hline 1981 & 2 & 4733840.9 & 10.2 & 265038.9 & 3.4 & 904558.1 & 0.3 & 0.128547 & 28.4 & 0 \\
\hline 1981 & 3 & 4609532.9 & 8.5 & 254464.7 & 4.7 & 1382255.5 & 1.5 & 0.135343 & 26.9 & 0 \\
\hline 1981 & 4 & 4899481.3 & 7.2 & 336935.9 & 3.3 & 1917030.0 & 0.1 & 0.144093 & 28.5 & 0 \\
\hline 1982 & 1 & 4803973.0 & 3.0 & 293736.2 & 21.9 & 679753.3 & 50.3 & 0.160927 & 32.8 & 0 \\
\hline 1982 & 2 & 4759293.7 & 0.5 & 297419.5 & 12.2 & 1251481.7 & 38.4 & 0.185496 & 44.3 & 1 \\
\hline 1982 & 3 & 4575880.7 & -0.7 & 248786.0 & -2.2 & 1666127.5 & 20.5 & 0.224169 & 65.6 & 1 \\
\hline 1982 & 4 & 4667298.1 & -4.7 & 290769.3 & -13.7 & 2040120.4 & 6.4 & 0.270317 & 87.6 & 1 \\
\hline 1983 & 1 & 4610574.3 & -4.0 & 195184.3 & -33.6 & 545229.0 & -19.8 & 0.342548 & 112.9 & 0 \\
\hline 1983 & 2 & 4528625.8 & -4.8 & 195896.4 & -34.1 & 956097.8 & -23.6 & 0.398272 & 114.7 & 0 \\
\hline 1983 & 3 & 4387418.8 & -4.1 & 175191.3 & -29.6 & 1294831.2 & -22.3 & 0.450194 & 100.8 & 0 \\
\hline 1983 & 4 & 4624154.5 & -0.9 & 219456.5 & -24.5 & 1676492.1 & -17.8 & 0.506570 & 87.4 & 0 \\
\hline 1984 & 1 & 4761483.9 & 3.3 & 195527.5 & 0.2 & 453527.6 & -16.8 & 0.592500 & 73.0 & 0 \\
\hline 1984 & 2 & 4661403.7 & 2.9 & 202250.1 & 3.2 & 819568.2 & -14.3 & 0.668479 & 67.8 & 0 \\
\hline 1984 & 3 & 4592953.7 & 4.7 & 185885.6 & 6.1 & 1140712.7 & -11.9 & 0.735237 & 63.3 & 0 \\
\hline 1984 & 4 & 4754021.3 & 2.8 & 237108.1 & 8.0 & 1504023.5 & -10.3 & 0.812414 & 60.4 & 0 \\
\hline 1985 & 1 & 4842551.7 & 1.7 & 174571.8 & -10.7 & 480654.0 & 6.0 & 0.944253 & 59.4 & 0 \\
\hline 1985 & 2 & 4831686.9 & 3.7 & 185690.6 & -8.2 & 893166.5 & 9.0 & 1.036024 & 55.0 & 0 \\
\hline 1985 & 3 & 4647011.4 & 1.2 & 169764.5 & -8.7 & 1236594.5 & 8.4 & 1.145528 & 55.8 & 0 \\
\hline 1985 & 4 & 4859239.5 & 2.2 & 210076.0 & -11.4 & 1581716.9 & 5.2 & 1.304766 & 60.6 & 0 \\
\hline
\end{tabular}




\section{Cuadro A.8. Series históricas}

(cifras en millones de pesos a precios de 2003) (continúa)

\begin{tabular}{|c|c|c|c|c|c|c|c|c|c|c|}
\hline Año & Trimestre & Absolutos & \begin{tabular}{|c|}
$\begin{array}{c}\text { Variación } \\
\text { anual } \\
(\%)\end{array}$ \\
\end{tabular} & Absolutos & $\begin{array}{c}\begin{array}{c}\text { Variación } \\
\text { anual } \\
(\%)\end{array} \\
\end{array}$ & Absolutos & $\begin{array}{c}\begin{array}{c}\text { Variación } \\
\text { anual } \\
(\%)\end{array} \\
\end{array}$ & Índice & $\begin{array}{c}\text { Variación } \\
\text { anual } \\
(\%)\end{array}$ & ELEC \\
\hline 1986 & 1 & 696606.4 & -3.0 & 197042.4 & 12.9 & 695238.0 & 44.6 & 1.573722 & 66.7 & 0 \\
\hline 1986 & 2 & 4810679.1 & -0.4 & 183232.5 & -1.3 & 1216446.8 & 36.2 & 1.833791 & 77.0 & 0 \\
\hline 1986 & 3 & 4426693.7 & -4.7 & 158955.5 & -6.4 & 1556761.0 & 25.9 & 2.193570 & 91.5 & 0 \\
\hline 1986 & 4 & 4655951.6 & -4.2 & 204666.5 & -2.6 & 1877314.0 & 18.7 & 2.650116 & 103.1 & 0 \\
\hline 1987 & 1 & 4648884.8 & -1.0 & 182465.2 & -7.4 & 784206.4 & 12.8 & 3.295423 & 109.4 & 0 \\
\hline 1987 & 2 & 4820702.9 & 0.2 & 158853.2 & -13.3 & 1280933.4 & 5.3 & 4.113357 & 124.3 & 0 \\
\hline 1987 & 3 & 4554345.4 & 2.9 & 154749.1 & -2.6 & 1570034.2 & 0.9 & 5.137413 & 134.2 & 0 \\
\hline 1987 & 4 & 4886198.1 & 4.9 & 167583.1 & -18.1 & 1785258.2 & -4.9 & 6.582348 & 148.4 & 0 \\
\hline 1988 & 1 & 4768290.4 & 2.6 & 134934.0 & -26.0 & 507370.2 & -35.3 & 9.142840 & 177.4 & 0 \\
\hline 1988 & 2 & 4872703.9 & 1.1 & 160127.6 & 0.8 & 928060.1 & -27.5 & 10.190871 & 147.8 & 1 \\
\hline 1988 & 3 & 4560000.1 & 0.1 & 127640.8 & -17.5 & 1359150.7 & -13.4 & 10.652474 & 107.4 & 1 \\
\hline 1988 & 4 & 4951 & 1.3 & 159512.0 & -4.8 & 1921 & 7.6 & 26 & 66.8 & 1 \\
\hline 1989 & 1 & 4906652.0 & 2.9 & 148673.9 & 10.2 & 443095.6 & -12.7 & 11.599746 & 26.9 & 0 \\
\hline 1989 & 2 & 5103243.5 & 4.7 & 140995.9 & -11.9 & 868262.1 & -6.4 & 12.069142 & 18.4 & 0 \\
\hline 1989 & 3 & 4824586.4 & 5.8 & 124661.3 & -2.3 & 1287278.7 & -5.3 & 12.4 & 17.0 & 0 \\
\hline 1989 & 4 & 5104635.6 & 3.1 & 170541.3 & 6.9 & 1793427.1 & -6.6 & 13.031742 & 18.7 & 0 \\
\hline 1990 & 1 & 5119608.0 & 4.3 & 137353.6 & -7.6 & 348130.8 & -21.4 & 7703 & 23.5 & 0 \\
\hline 1990 & 2 & 5309633.7 & 4.0 & 135143.5 & -4.2 & 673134.7 & -22.5 & 15.104536 & 25.2 & 0 \\
\hline 1990 & 3 & 5063047.8 & 4.9 & 127922.9 & 2.6 & 976004.3 & -24.2 & 15.947343 & 28.0 & 0 \\
\hline 1990 & 4 & 5478830.4 & 7.3 & 178352.0 & 4.6 & 1342684.9 & -25.1 & 16.888624 & 29.6 & 0 \\
\hline 1991 & 1 & 5314150.1 & 3.8 & 157092.0 & 14.4 & 290591.4 & -16.5 & 18.125111 & 26.5 & 0 \\
\hline 1991 & 2 & 5608968.2 & 5.6 & 154131.0 & 14.0 & 571776.9 & -15.1 & 18.780975 & 24.3 & 0 \\
\hline 1991 & 3 & 5234160.1 & 3.4 & 144066.5 & 12.6 & 852053.8 & -12.7 & 383 & 21.0 & 0 \\
\hline 1991 & 4 & 5697722.8 & 4.0 & 191217.9 & 7.2 & 1187019.2 & -11.6 & 20.176506 & 19.5 & 0 \\
\hline 1992 & 1 & 5563435.2 & 4.7 & 155052.3 & -1.3 & 234456.5 & -19.3 & 21.269918 & 17.4 & 0 \\
\hline 1992 & 2 & 5738305.7 & 2.3 & 160475.2 & 4.1 & 465676.4 & -18.6 & 21.833591 & 16.3 & 0 \\
\hline 1992 & 3 & 5469093.2 & 4.5 & 143300.1 & -0.5 & 698792.6 & -18.0 & 22.273671 & 15.4 & 0 \\
\hline 1992 & 4 & 5858075.0 & 2.8 & 192497.5 & 0.7 & 992689.1 & -16.4 & 22.843104 & 13.2 & 0 \\
\hline 1993 & 1 & 5732746.1 & 3.0 & 147032.0 & -5.2 & 275380.3 & 17.5 & 23.585539 & 10.9 & 0 \\
\hline 1993 & 2 & 5802801.8 & 1.1 & 150150.3 & -6.4 & 551441.1 & 18.4 & 24.013802 & 10.0 & 0 \\
\hline 1993 & 3 & 5857838.6 & 7.1 & 143894.7 & 0.4 & 830401.7 & 18.8 & 24.411952 & 9.6 & 0 \\
\hline 1993 & 4 & 6092879.1 & 4.0 & 190203.8 & -1.2 & 1190313.9 & 19.9 & 24.811757 & 8.6 & 0 \\
\hline 1994 & 1 & 5896049.7 & 2.8 & 177321.7 & 20.6 & 291745.1 & 5.9 & 25.297292 & 7.3 & 0 \\
\hline 1994 & 2 & 6138930.2 & 5.8 & 168952.8 & 12.5 & 586027.3 & 6.3 & 25.676814 & 6.9 & 1 \\
\hline 1994 & 3 & 6153162.6 & 5.0 & 168078.6 & 16.8 & 883913.9 & 6.4 & 26.060315 & 6.8 & 1 \\
\hline 1994 & 4 & 6424823.8 & 5.4 & 214582.6 & 12.8 & 1264821.6 & 6.3 & 26.533142 & 6.9 & 1 \\
\hline 1995 & 1 & 5826003.7 & -1.2 & 167753.3 & -5.4 & 341137.8 & 16.9 & 29.093440 & 15.0 & 0 \\
\hline 1995 & 2 & 5600037.0 & -8.8 & 136906.1 & -19.0 & 589130.2 & 0.5 & 34.347491 & 33.8 & 0 \\
\hline 1995 & 3 & 5691934.6 & -7.5 & 127935.8 & -23.9 & 839105.6 & -5.1 & 36.916484 & 41.7 & 0 \\
\hline 1995 & 4 & 5962217.0 & -7.2 & 169315.6 & -21.1 & 1143746.6 & -9.6 & 39.458032 & 48.7 & 0 \\
\hline
\end{tabular}


232 ECONOMÍA: TEORÍA Y PRÁCTICA • Nueva Época, número 40, enero-junio 2014

\section{Cuadro A.8. Series históricas}

(cifras en millones de pesos a precios de 2003)

(continúa)

\begin{tabular}{|c|c|c|c|c|c|c|c|c|c|c|}
\hline Año & Trimestre & Absolutos & \begin{tabular}{|c|} 
Variación \\
anual \\
$(\%)$
\end{tabular} & Absolutos & $\begin{array}{c}\text { Variación } \\
\text { anual } \\
(\%)\end{array}$ & Absolutos & \begin{tabular}{|c|} 
Variación \\
anual \\
$(\%)$
\end{tabular} & Índice & $\begin{array}{c}\text { Variación } \\
\text { anual } \\
(\%)\end{array}$ & $E L E C$ \\
\hline 1996 & 1 & 5891226.8 & 1.1 & 138178.6 & -17.6 & 308327.1 & -9.6 & 43.059767 & 48.0 & 0 \\
\hline 1996 & 2 & 5955682.2 & 6.4 & 133690.0 & -2.3 & 587539.6 & -0.3 & 46.070994 & 34.1 & 0 \\
\hline 1996 & 3 & 6073486.7 & 6.7 & 127910.2 & 0.0 & 859881.5 & 2.5 & 48.189986 & 30.5 & 0 \\
\hline 1996 & 4 & 6427559.4 & 7.8 & 166118.4 & -1.9 & 1194010.4 & 4.4 & 50.560993 & 28.1 & 0 \\
\hline 1997 & 1 & 6152807.2 & 4.4 & 147206.8 & 6.5 & 313269.5 & 1.6 & 54.039136 & 25.5 & 0 \\
\hline 1997 & 2 & 6492761.7 & 9.0 & 143769.1 & 7.5 & 617659.4 & 5.1 & 55.880309 & 21.3 & 0 \\
\hline 1997 & 3 & 6569860.1 & 8.2 & 140887.9 & 10.1 & 919771.8 & 7.0 & 57.445819 & 19.2 & 0 \\
\hline 1997 & 4 & 6898432.2 & 7.3 & 183726.2 & 10.6 & 1298806.9 & 8.8 & 59.268263 & 17.2 & 0 \\
\hline 1998 & 1 & 6665227.0 & 8.3 & 153484.5 & 4.3 & 299457.5 & -4.4 & 62.305640 & 15.3 & 0 \\
\hline 1998 & 2 & 6779735.0 & 4.4 & 154170.0 & 7.2 & 591297.7 & -4.3 & 64.333579 & 15.1 & 0 \\
\hline 1998 & 3 & 6891651.8 & 4.9 & 150438.0 & 6.8 & 876817.8 & -4.7 & 66.414856 & 15.6 & 0 \\
\hline 1998 & 4 & 7072200.6 & 2.5 & 188764.0 & 2.7 & 1217597.9 & -6.3 & 69.678528 & 17.6 & 0 \\
\hline 1999 & 1 & 6833076.8 & 2.5 & 168680.0 & 9.9 & 309977.0 & 3.5 & 73.894110 & 18.6 & 0 \\
\hline 1999 & 2 & 6997209.3 & 3.2 & 160832.5 & 4.3 & 615831.2 & 4.1 & 75.833117 & 17.9 & 0 \\
\hline 1999 & 3 & 7148246.5 & 3.7 & 164732.6 & 9.5 & 924160.0 & 5.4 & 77.357804 & 16.5 & 0 \\
\hline 1999 & 4 & 7410151.1 & 4.8 & 238210.3 & 26.2 & 1314698.3 & 8.0 & 79.223401 & 13.7 & 0 \\
\hline 2000 & 1 & 7298852.8 & 6.8 & 187288.4 & 11.0 & 342211.3 & 10.4 & 81.689431 & 10.5 & 0 \\
\hline 2000 & 2 & 7467037.7 & 6.7 & 199267.0 & 23.9 & 686122.2 & 11.4 & 83.069210 & 9.5 & 1 \\
\hline 2000 & 3 & 7604284.6 & 6.4 & 191371.9 & 16.2 & 1034542.8 & 11.9 & 84.338085 & 9.0 & 1 \\
\hline 2000 & 4 & 7711444.3 & 4.1 & 242154.2 & 1.7 & 1473209.1 & 12.1 & 86.285155 & 8.9 & 1 \\
\hline 2001 & 1 & 7310622.3 & 0.2 & 200625.3 & 7.1 & 335816.1 & -1.9 & 87.780498 & 7.5 & 0 \\
\hline 2001 & 2 & 7424984.9 & -0.6 & 203572.4 & 2.2 & 676955.7 & -1.3 & 88.781133 & 6.9 & 0 \\
\hline 2001 & 3 & 7483060.8 & -1.6 & 203823.1 & 6.5 & 1029308.6 & -0.5 & 89.385278 & 6.0 & 0 \\
\hline 2001 & 4 & 7576346.9 & -1.8 & 248460.6 & 2.6 & 1476373.0 & 0.2 & 90.791207 & 5.2 & 0 \\
\hline 2002 & 1 & 7110694.3 & -2.7 & 235256.6 & 17.3 & 356685.3 & 6.2 & 91.945813 & 4.7 & 0 \\
\hline 2002 & 2 & 7523677.2 & 1.3 & 227794.4 & 11.9 & 718828.8 & 6.2 & 93.019291 & 4.8 & 0 \\
\hline 2002 & 3 & 7517032.6 & 0.5 & 224275.2 & 10.0 & 1088030.9 & 5.7 & 94.077972 & 5.2 & 0 \\
\hline 2002 & 4 & 7670055.4 & 1.2 & 275964.6 & 11.1 & 1559232.0 & 5.6 & 95.641582 & 5.3 & 0 \\
\hline 2003 & 1 & 7367050.4 & 3.6 & 245340.0 & 4.3 & 380143.4 & 6.6 & 96.946926 & 5.4 & 0 \\
\hline 2003 & 2 & 7539706.2 & 0.2 & 246356.2 & 8.1 & 771229.2 & 7.3 & 97.427007 & 4.7 & 0 \\
\hline 2003 & 3 & 7535289.5 & 0.2 & 243808.2 & 8.7 & 1174856.6 & 8.0 & 97.905841 & 4.1 & 0 \\
\hline 2003 & 4 & 7781167.4 & 1.4 & 305319.6 & 10.6 & 1685211.8 & 8.1 & 99.441412 & 4.0 & 0 \\
\hline 2004 & 1 & 7620258.2 & 3.4 & 264417.9 & 7.8 & 393756.0 & 3.6 & 101.137114 & 4.3 & 0 \\
\hline 2004 & 2 & 7823421.3 & 3.8 & 274445.4 & 11.4 & 799114.8 & 3.6 & 101.603798 & 4.3 & 0 \\
\hline 2004 & 3 & 7871310.1 & 4.5 & 273685.7 & 12.3 & 1211495.4 & 3.1 & 102.595424 & 4.8 & 0 \\
\hline 2004 & 4 & 8133297.8 & 4.5 & 324750.9 & 6.4 & 1728712.0 & 2.6 & 104.750333 & 5.3 & 0 \\
\hline 2005 & 1 & 7773298.6 & 2.0 & 295354.9 & 11.7 & 414126.3 & 5.2 & 105.586813 & 4.4 & 0 \\
\hline 2005 & 2 & 8117334.1 & 3.8 & 295847.2 & 7.8 & 839567.8 & 5.1 & 106.186213 & 4.5 & 0 \\
\hline 2005 & 3 & 8141697.9 & 3.4 & 292932.4 & 7.0 & 1279402.1 & 5.6 & 106.671277 & 4.0 & 0 \\
\hline 2005 & 4 & 8424010.4 & 3.6 & 351893.2 & 8.4 & 1841075.8 & 6.5 & 107.996872 & 3.1 & 0 \\
\hline
\end{tabular}




\section{Cuadro A.8. Series históricas}

(cifras en millones de pesos a precios de 2003)

(concluye)

\begin{tabular}{|c|c|c|c|c|c|c|c|c|c|c|}
\hline Año & Trimestre & Absolutos & \begin{tabular}{|c|}
$\begin{array}{c}\text { Variación } \\
\text { anual } \\
(\%)\end{array}$ \\
\end{tabular} & Absolutos & $\begin{array}{c}\text { Variación } \\
\text { anual } \\
(\%)\end{array}$ & Absolutos & $\begin{array}{c}\text { Variación } \\
\text { anual } \\
(\%)\end{array}$ & Índice & $\begin{array}{c}\text { Variación } \\
\text { anual } \\
(\%)\end{array}$ & ELEC \\
\hline 2006 & 1 & 8252084.5 & 6.2 & 322440.0 & 9.2 & 458909.6 & 10.8 & 109.491321 & 3.7 & 0 \\
\hline 2006 & 2 & 8546940.9 & 5.3 & 337036.9 & 13.9 & 935522.3 & 11.4 & 109.505028 & 3.1 & 1 \\
\hline 2006 & 3 & 8563555.0 & 5.2 & 332405.7 & 13.5 & 1419894.0 & 11.0 & 110.449300 & 3.5 & 1 \\
\hline 2006 & 4 & 8765311.5 & 4.1 & 399937.0 & 13.7 & 2031415.2 & 10.3 & 112.473052 & 4.1 & 1 \\
\hline 2007 & 1 & 8502889.8 & 3.0 & 359547.1 & 11.5 & 485562.3 & 5.8 & 113.980585 & 4.1 & 0 \\
\hline 2007 & 2 & 8798065.8 & 2.9 & 358982.9 & 6.5 & 991055.2 & 5.9 & 113.856904 & 4.0 & 0 \\
\hline 2007 & 3 & 8859918.4 & 3.5 & 359104.9 & 8.0 & 1503997.5 & 5.9 & 114.852580 & 4.0 & 0 \\
\hline 2007 & 4 & 9079671.3 & 3.6 & 423730.9 & 5.9 & 2155391.8 & 6.1 & 116.758882 & 3.8 & 0 \\
\hline 2008 & 1 & 8682295.4 & 2.1 & 378079.5 & 5.2 & 541442.0 & 11.5 & 118.417199 & 3.9 & 0 \\
\hline 2008 & 2 & 9018001.6 & 2.5 & 374714.0 & 4.4 & 1094319.1 & 10.4 & 119.454933 & 4.9 & 0 \\
\hline 2008 & 3 & 8977691.9 & 1.3 & 378561.7 & 5.4 & 1651834.2 & 9.8 & 121.146896 & 5.5 & 0 \\
\hline 2008 & 4 & 8982131.9 & -1.1 & 465848.1 & 9.9 & 2351620.9 & 9.1 & 123.976597 & 6.2 & 0 \\
\hline $2009^{P}$ & 1 & 8034689.9 & -7.5 & 424576.1 & 12.3 & 546521.1 & 0.9 & 125.729934 & 6.2 & 0 \\
\hline 2009 & 2 & 8138850.7 & -9.7 & 420004.3 & 12.1 & 1106805.0 & 1.1 & 126.577318 & 6.0 & 0 \\
\hline 2009 & 3 & 8472712.4 & -5.6 & 412018.6 & 8.8 & 1683805.9 & 1.9 & 127.369559 & 5.1 & 0 \\
\hline 2009 & 4 & 8788463.2 & -2.2 & 490309.4 & 5.3 & 2423911.8 & 3.1 & 128.904819 & 4.0 & 0 \\
\hline 2010 & 1 & 8399898.7 & 4.5 & 453429.4 & 6.8 & 562643.7 & 3.0 & 131.706170 & 4.8 & 0 \\
\hline 2010 & 2 & 8774558.9 & 7.8 & 439102.1 & 4.5 & 1148145.9 & 3.7 & 131.590278 & 4.0 & 0 \\
\hline 2010 & 3 & 8928726.9 & 5.4 & 446261.1 & 8.3 & 1751585.1 & 4.0 & 132.044500 & 3.7 & 0 \\
\hline 2010 & 4 & 9176856.9 & 4.4 & 516012.7 & 5.2 & 2507505.4 & 3.4 & 134.381036 & 4.2 & 0 \\
\hline 2011 & 1 & 8769238.5 & 4.4 & 465785.2 & 2.7 & 585810.5 & 4.1 & 136.267169 & 3.5 & 0 \\
\hline 2011 & 2 & 9052992.2 & 3.2 & 467400.8 & 6.4 & 1197358.0 & 4.3 & 135.926986 & 3.3 & 0 \\
\hline 2011 & 3 & 9332160.4 & 4.5 & 471288.7 & 5.6 & 1825371.5 & 4.2 & 136.492603 & 3.4 & 0 \\
\hline 2011 & 4 & 9514636.7 & 3.7 & 548939.6 & 6.4 & 2609809.4 & 4.1 & 139.084864 & 3.5 & 0 \\
\hline
\end{tabular}

Fuentes: *La serie trimestral $19599_{1}-19799_{4}$ se estimó con base en el coeficiente de estacionalidad que resultó de la misma serie con cifras observadas del periodo 1980 $-2011_{4}$. Datos de 1960-1980, SCNM $1980=100$, INEGI y 1981-2011 PIB trimestral $2003_{3}=100$, BIE de INEGI.

** La serie trimestral $1959_{1}-1985_{3}$ se estimó con base en el coeficiente de estacionalidad que resultó de la misma serie con cifras observadas del periodo $1985_{4}-2011_{4}$. Datos de 1960-1983, Anuarios Estadísticos de los Estados Unidos Mexicanos 1962-63, 1968-69, 1972-74, 1977-1978 y 1981, INEGI. De 1983-2011, Banxico.

$* * *$ La serie trimestral $1959_{1}-2011_{4}$ se estimó con base en el coeficiente de estacionalidad que resultó de la serie del gasto presupuestal del sector público que reporta Banxico con cifras observadas del periodo $1977_{3}-2011_{4}$ porque sus datos anuales se asemejan a los datos de los egresos y esta serie no tiene cifras trimestrales. Para 2011 se consideraron las cifras que reporta Banxico porque no hay datos publicados de los egresos del sector público presupuestario de la SHCP. Datos de 1960-1979, Anuarios Estadísticos de los Estados Unidos Mexicanos 1962-63, 1968-69, 1972-74, 1977-1978 y 1981, SHCP. De 1977-2011, Banxico. De 1980-2010, Sistema de Finanzas Públicas, SHCP.

**** De 1960-1975, Índice de precios al mayoreo de la Ciudad de México, INEGI. De 1976-2011, Banxico.

P Cifras preliminares para el PIB. 


\section{REFERENCIAS BIBLIOGRÁFICAS}

Alesina, Alberto, y Roubini, Nouriel (1992), "Political Cycles in OECD Economies", Review of Economic Studies, 59 (4), pp. 663-688.

Bonilla, Claudio A., y Gatica, Leonardo A. (2005), "Economía política neoclásica y la América Latina, una mirada a la bibliografía", El Trimestre Económico, 72 (285), enero-marzo, pp. 179-211.

Buchanan, James M. (1960), La Scienza delle Finanze: The Italian Tradition in Public Finance. Fiscal Theory and Political Economic, Chapel Hill, University of North Carolina Press.

Centro de Estudios de las Finanzas Públicas (2006), "Cuenta de la Hacienda Pública Federal 2006. Análisis Funcional Programático del Sector Público Presupuestario", en Cámara de Diputados. Consultado el 8 de octubre de 2009 en: http:// www3.diputados.gob.mx/camara/001_diputados/006_centros_de_estudio/02 centro_de_estudios_de_finanzas_publicas_1/006_presupuesto_y_gasto/04_ cuenta_publica.

Constitución Política de los Estados Unidos Mexicanos (1917) [en línea]. Consultado el 15 de julio de 2013 en: http://www.diputados.gob.mx/LeyesBiblio/pdf/1.pdf.

Flores, Daniel (2007), "Elecciones y ciclos económicos en México", El Trimestre Económico, 74 (294), abril-junio, pp. 467-474.

Gámez, Cesáreo (2004), "El ciclo político oportunista y la economía mexicana (1980-2004)", ponencia al II Coloquio Predoctoral Latinoamericano, xxxix Asamblea Anual de CLADEA realizado en Puerto Plata, Santo Domingo, 19 y 20 de octubre [en línea]. Consultado el 20 de mayo de 2008 en: http://www.revistaleadership. com/cladea/doctoral/coloquio_II/GAMEZCESAREO.pdf.

Gámez, Cesáreo, y Amarillas, Víctor A. (2011), "Política económica o economía política: el ciclo político presupuestal en México", Cofactor, 2 (3) [en línea]. Consultado el 25 de junio de 2013 en: http://portal2.edomex.gob.mx/cofactor/numeros anteriores/economia_politica_migracion_politica_social/ciclo_politico_ presupuestal_mexico/groups/public/documents/edomex_archivo/cofactor_pdf_ art4cof3.pdf.

Grier, Kevin B., y Hernández-Trillo, Fausto (2004), “The Real Exchange Rate, Process and its Real Effects: The Cases of Mexico and the USA", Journal of Applied Economics, 7 (1), mayo, pp. 1-25.

Gujarati, Damodar N. (2010), Econometría, 5a. edición, México, McGraw Hill.

Hibbs, Douglas A. Jr., (1977), "Political Parties and Macroeconomic Policy", The American Political Science Review, 71 (4), pp. 1467-1487. 
Hotelling, Harold (1929), “Stability in Competition”, Economic Journal, 39 (153), marzo, pp. 41-57.

Kalecki, Michal (1943), "Political Aspects of Full Employment”, Political Quarterly [en línea]. Consultado el 30 de junio de 2013 en: http://www.modernmoneyandpublic purpose.com/uploads/1/2/5/3/12534585/political-aspects-of-full-employment. pdf.

Lindahl, Erik (1958), “Just taxation - A Positive Solution”. en Richard Musgrave y Alan T. Pecock (eds.), Classics in the theory of public finance. Nueva York, St. Martin's.

Magaloni, Beatriz (2000), "Institutions, Political Opportunism and Macroeconomic Cycles: México 1970-1998”, documento de trabajo, Stanford University, marzo [en línea].Consultado el 25 de mayo de 2007 en: http://www.stanford.edu/ group/sshi/Conferences/1999-2000/SpringPapers/magaloni.pdf.

Massad, Carlos A., y Pattillo, Guillermo (2000), Macroeconomía en un mundo interdependiente, Chile, McGraw Hill Interamericana.

Nordhaus, William D. (1975), “The Political Business Cycle”, Review of Economic Studies, 42 (2), pp. 169-190.

Samuelson, Paul A. (1954), "The Pure Theory of Public Expenditure", The Review of Economics and Statistics, 36 (4), noviembre, pp. 387-389.

Téllez, Francisco R. (1997), “Análisis del ciclo político de la economía en México, 19701994. Una perspectiva desde la teoría de la elección pública”, tesis de licenciatura en Economía, Facultad de Economía de la UNAM.

Tufte, Edward R. (1978), Political Control of the Economy, Princenton, NJ, Princenton University Press. 